\title{
A COMPARISON OF FATIGUE DURING CARDIOCEREBRAL RESUSCITATION WITH DIFFERENT COMPRESSION RATES AMONG LAYPERSON AND PROFESSIONAL RESCUERS
}

\author{
A Thesis \\ presented to \\ the Faculty of California Polytechnic State University, \\ San Luis Obispo
}

\author{
In Partial Fulfillment \\ of the Requirements for the Degree \\ Master of Science in Kinesiology \\ by \\ Christopher Cassidy
}

August 2014 
(C) 2014

Christopher Cassidy

ALL RIGHTS RESERVED 
TITLE:

AUTHOR:

DATE SUBMITTED:

COMMITTEE CHAIR:

COMMITTEE MEMBER:

COMMITTEE MEMBER:

COMMITTEE MEMBER:
A Comparison of Fatigue During Cardiocerebral

Resuscitation with Different Compression Rates Among Layperson and Professional Rescuers

Christopher Cassidy

August 2014

Steven C. Davis, Ph.D., R.C.E.P.

Professor of Kinesiology

Patricia Hosegood Martin, Ed.D.

Associate Human Resources Director

Kellie Green Hall, Ph.D.

Professor of Kinesiology

Greg Hall, M.S.

Instructor of Kinesiology 


\begin{abstract}
A Comparison of Fatigue During Cardiocerebral Resuscitation with Different Compression Rates Among Layperson and Professional Rescuers
\end{abstract}

\title{
Christopher Cassidy
}

Quality chest compressions during Cardiopulmonary Resuscitation (CPR) are vital to maintaining adequate perfusion of oxygenated blood to the organs of the body necessary to sustain life. Over the years, the compression rate recommended in Basic Life Support (BLS)/ Advanced Cardiac Life Support (ACLS) protocols for the best possible outcome has risen, and with that increase there are questions regarding rescuer fatigue and the effectiveness of compressions. Laypersons and professional rescuers, answering to an emergency, both maintain continuous chest compressions until advanced life support arrives. Depending on the location, this arrival time would most likely be longer than the two minute standard time given to rotate rescuers before fatigue compromises compression performance. The objective of this research was to investigate the level of rescuer fatigue associated with continuous compressions, varying compression rates, physical fitness, gender, and among laypersons versus professional rescuers.

Sixty-one participants performed uninterrupted chest compressions on a manikin for 15 minutes. Before performing compressions, physical fitness was evaluated using a YMCA bench press for an assessment of upper body strength/endurance and a 3-minute step test to evaluate aerobic fitness. Subjects performed two trials at compression rates of $\geq 80$ and $\geq 100$ per minute with a 5 -minute rest between trials. 
Male professional rescuers had greater strength/endurance, and thus were able to perform compressions for a longer period of time compared to their female counterparts. Compression duration and the YMCA Bench Press test score were significantly correlated $(\mathrm{p}=.0135)$. Cardiocerebral resuscitation providers should maintain an adequate upper body strength, particularly if they may be required to perform continuous chest compressions at high rates for more than a few minutes.

Keywords: Continuous compressions, compression rates, Emergency Medical Services (EMS), response time, cardiac perfusion, and patient survival. 


\section{ACKNOWLEDGMENTS}

I would like to acknowledge Dr. Steven C. Davis for dedicating his time and energy into turning a research idea into a great experience. I would like to thank my committee members Dr. Patricia Hosegood Martin, Dr. Kellie Green Hall, and Mr.

Greg Hall for their support and expertise throughout the writing process. Lastly, I want to recognize my parents, Charles and Sandra Cassidy, and dedicate the sum of my work to their unrelenting belief in my ability to achieve. 


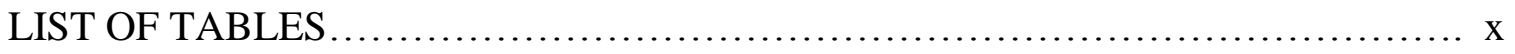

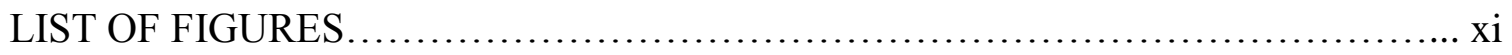

\section{CHAPTER}

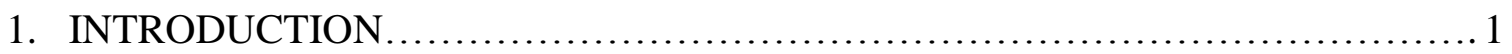

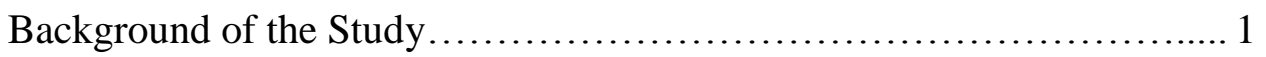

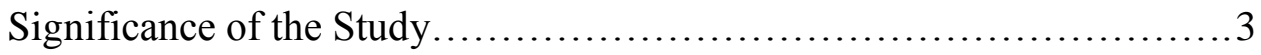

Statement of the Problem................................................... 5

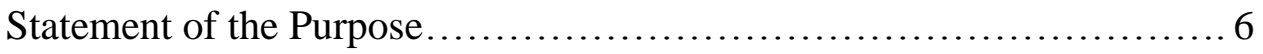

Delimitations..................................................... 6

Limitations ........................................................ 6

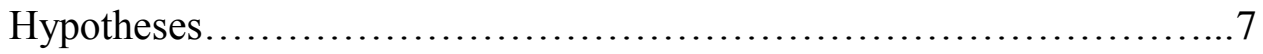

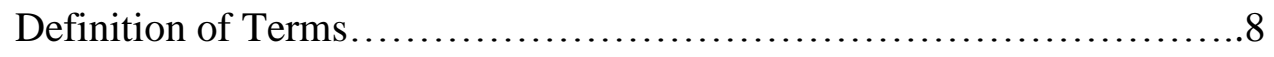

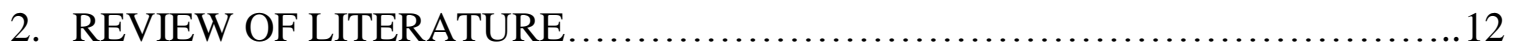

Method................................................................

Discussion..................................................... 12

3. METHODS AND PROCEDURES .................................................. 35

Restatement of the Purpose ........................................... 35

Subjects......................................................... 35

Experimenters..................................................... 36

Chest Depth and Force Measurements...................................36

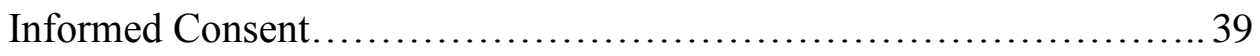


Par-Q and Targeted History.

YMCA Bench Press Test.

YMCA Step Test............................................. 41

Chest Compression Tests..................................... 42

Preston Manikin............................................. 43

Statistical Analysis......................................... 47

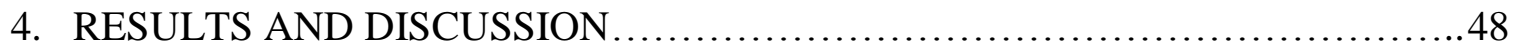

Results....................................................... 48

Subjects..................................................48

Fitness Tests...............................................48

Chest Compression Tests....................................... 51

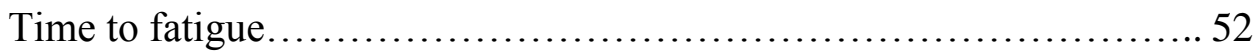

Ratings of perceived exertion...................................54

Heart rate....................................................55

Discussion.................................................. 59

Interpretation of Results.......................................59

5. SUMMARY, CONCLUSIONS, AND RECOMMENDATIONS.....................64

Summary.............................................. 64

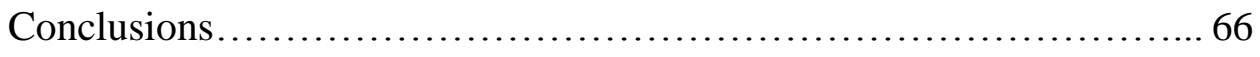

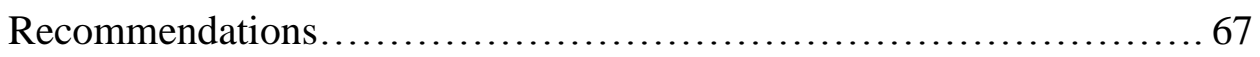

Future Research............................................67

Performance of Cardiocerebral Resuscitation...................... 67 


\section{APPENDICES}

A. Human Subjects Protocol Approval Form................................ 78

B. Informed Consent Form............................................ 84

C. Physical Activity Readiness Questionnaire (PAR-Q) ........................86

D. YMCA Bench Press Test.............................................. 87

E. YMCA 3-Minute Step Test......................................... 90

F. Guidelines for CPR .................................................. 93 


\section{LIST OF TABLES}

Table

1. A Literature Review Summary of Compression Rates, Ratios, and Findings. The Chart Headings Show the Three Different Compression-to-Ventilation Ratios (15:2, 30:2, 50:2) and Continuous Compressions (CC). The Heading (min) is the Duration of the Compression Trial. The Heading (rate) Refers

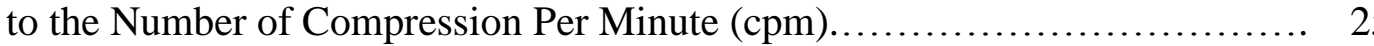

2. Characteristics of Subjects........................................... 49

3. YMCA Fitness Test Scores............................................. 50

4. RPE Tabulated for 5, 10, and 15 Minutes at $\geq 80$ and $\geq 100 \mathrm{cpm} \ldots \ldots \ldots \ldots \ldots$

5. HR Tabulated for 5, 10, and 15 Minutes at $\geq 80$ and $\geq 100 \mathrm{cpm} \ldots \ldots \ldots \ldots \ldots . . . \ldots 7$ 


\section{LIST OF FIGURES}

Figure

Page

1. Differences in Forces Between Chest Compressions Performed on Both Manikins (A, B) Used in This Study. The Line Within the Box Indicates the Mean Compression Force (Force on the Z Axis in Newtons, FzN) for Each Week for Either Manikin A or B. The Lines Extending From the Top and Bottom of Each Box is Illustrating the Standard Error for Each Week for Either Manikin A or B.

2. Testing Procedures...................................................... 38

3. YMCA Bench Press Test............................................. 40

4. YMCA 3-Minute Step Test.............................................. 41

5. RPE Scale......................................................... 42

6. Light Biofeedback on Shoulder of CPR Manikin........................... 43

7. Chest Compressions on Manikin...................................... 45

8. Test Procedures: Controlling for Order Effect............................ 46

9. Bench Press Percentile Versus Reaching 15 Minutes at $\geq 80 \mathrm{cpm}$. On the $Y$ Axis, the Graph is Split Using (0) for Not Making it to 15 Minutes and (1) for Making it to 15 Minutes

10. Bench Press Percentile Versus Reaching 15 Minutes at $\geq 100 \mathrm{cpm}$. On the $Y$ Axis, the Graph is Split Using (0) for Not Making it to 15 Minutes and (1) for Making it to 15 Minutes

11. Average RPE at 5, 10, and 15 minutes Versus M/F Subjects................ 56

12. Average RPE at 5, 10, and 15 minutes Versus P/L Subjects................. 56

13. Average $\mathrm{HR}$ at 5, 10, and 15 minutes Versus M/F Subjects.................. 58

14. Average $\mathrm{HR}$ at 5, 10, and 15 minutes Versus P/L Subjects................... 58

15. Reasons for Discontinuing Chest Compressions Before Completing 15 Minutes. 


\section{Chapter 1}

\section{INTRODUCTION}

\section{Background of the Study}

According to the Centers for Disease Control and Prevention (CDC) heart disease is the leading cause of death in the United States of America (U.S.A.), with approximately 600,000 cardiac deaths per year (CDC, 2014). According to the American Heart Association (AHA, 2013), "Nearly 383,000 out-of-hospital sudden cardiac arrests occur annually, and 88 percent of cardiac arrests occur at home” (CPR Statistics, Why Learn CPR?). While electrical therapy is more efficacious in restoring normal cardiac rhythm and cardiac output in patients in ventricular fibrillation or pulseless ventricular tachycardia, cardiopulmonary resuscitation $(\mathrm{CPR})$ is the first aid therapy used until a monitor-defibrillator or automated external defibrillator (AED) can be used for treatment of these rhythms. Cardiopulmonary resuscitation is also the appropriate technique when cardiac arrest is related to a non-shockable heart rhythm (e.g., asystole), or among laypersons lacking the training or the equipment to administer electrical therapy. Effective CPR administered immediately upon cardiac arrest results in survival of approximately $8 \%$ of arrest victims outside of the hospital (AHA, 2011).

Compression rate depends on the number of compressions delivered and the amount of time they are performed without interruption. Over the years, the frequency of compressions thought needed for the best possible patient outcome has risen. This increase in the compressions per minute allows for improved arterial pressure (hemodynamics) and maintains the delivery of oxygenated blood to the vital organs. According to Berg, Hemphill, Abella, Aufderheide, Cave, Hazinski, Lerner, Rea, Sayre, 
and Swor (2010), "The number of chest compressions delivered per minute is an important determinant of return of spontaneous circulation (ROSC) and neurologically intact survival” (pp. 5685-5705).

Ventilations during CPR have been de-emphasized (in emergency situations) over the years to make time for improved arterial perfusion (Kern, Hilwig, Berg, Sanders, \& Ewy, 2002). Relying more on compressions utilizes residual volumes and chest release to draw in what oxygen is needed. A study by Kern et al. (2002) looked at the hemodynamic compromise associated with discontinuing chest compressions to administer ventilations. Researchers found that the average time to perform mouth-tomouth was 16 seconds, which accounted for $60 \%$ of the resuscitation time.

Layperson and professional rescuers, responding to an emergency, both maintain continuous chest compressions until further life support can be established. Depending on the location, the time required for Advanced Life Support (ALS) to arrive will probably be longer than the standard two minutes given to rotate rescuers before compression performance is compromised by fatigue (Manders \& Geijsel, 2009). As a single rescuer, the physical inability to maintain adequate chest compressions, and supply needed oxygen over time, may compromise patient survival before ALS providers have the opportunity to provide care.

Since 2005, according to the American Heart Association (2013) the protocol for administering CPR has changed from performing 15 chest compressions and 2 breaths (15:2) to 30 compressions and 2 breaths (30:2). More recently, according to the Highlights of the 2010 AHA Guidelines for CPR, emphasis has been placed on the lone rescuer beginning CPR with compressions and delivering at least 100 compressions per 
minute while minimizing interruptions (AHA, 2011). A study by Betz, Callaway, Hostler, and Rittenberger (2008) stated that this increase in chest compressions may present a physical burden to the provider due to the additional exertion that is required. Significance of the Study

Cardiac arrest, as with acute heart attack, is fatal unless circulation can be sustained by a rescuer until the heart resumes its natural pumping action. For many years the first aid treatment for cardiac arrest has been "cardiopulmonary resuscitation" (CPR), which involves both chest compressions and artificial ventilations. In effect, rescuers circulate the blood and breathe oxygen-rich atmospheric air into the lungs for the victim.

With increased research evidence the protocol for CPR continues to evolve. The trend is toward increased emphasis on more rapid chest compressions and decreased emphasis on artificial ventilations. The recommended compression frequency has increased from 80 compressions per minute to at least 100 compressions per minute. Ventilations are not considered essential as long as the blood is being circulated by good external chest compressions, though ventilations are still encouraged, particularly when there is more than one rescuer and supplemental oxygen is available.

Compressing the chest at the rate of at least 100 per minute to a recommended depth of 2 inches may be more fatiguing than the slower rate of 80 per minute. While $100 / \mathrm{min}$ is quite sustainable when this is performed in-hospital with multiple rescuers who can switch off every 2 minutes (Riera et al., 2006), there is some question about how long this can be sustained by single rescuers. It is hypothesized that single rescuers may not be able to sustain this level of exercise long enough for help to arrive, particularly when chest compressions are being sustained by older lay rescuers in rural settings. This 
study seeks to describe fatigue rates at $\geq 80 \mathrm{vs.} \geq 100$ compressions per minute (cpm), and verify the utility of the current CPR guidelines for single rescuers among laypersons versus professionals, men versus women of varying ages, and persons of varying chest strength/endurance and cardiorespiratory fitness.

A number of studies have examined professional rescuers (Hansen et al., 2012; Russo et al., 2011) and a few have involved layperson rescuers (McDonald, Heggie, Jones, Thorne, \& Hulme, 2013, Trowbridge et al., 2009), yet there is a lack of research surrounding how fatigue affects the quality of compressions in comparing both professional and layperson rescuers. This research highlights the possible need, in both professional and layperson CPR, for improved physical fitness in adapting to the increasing compression rates. This was designed to evaluate how much of an effect physical fatigue has on the quality of chest compressions. Many studies have evaluated the effects of continuous compressions on the quality of CPR (Ashton, McCluskey, Gwinnutt, \& Keenan, 2002; Ock, Kim, Chung, \& Kim, 2011). The evaluation of this dependent measure would give an accurate interpretation of rescuer fatigue and procedures following a sudden cardiac arrest emergency.

This study stands out further from previous studies examining rescuer fatigue due to the impact it could have on the practice of CPR nationwide. If significant data show the patient's chance of survival is related to the physical fitness of the rescuer, it may change protocols of the re-certification process already in place. This finding might induce those requiring CPR certification to uphold a higher physical fitness standard before beginning the re-certification process. Also, a better understanding of the relationship between layperson and professional rescuers allows for a more accurate 
determination of how often CPR should be practiced and possibly lead to a justification in providing an increase in rescue personnel to allow for decreased response times.

The findings from this study may indicate recommendations that might be significant to the rescuer in providing the best possible care to the victim of cardiac arrest. With emergency services response times varying with each call, it is undetermined how long a rescuer will have to perform CPR before ALS arrives. The AHA's Chain of Survival protocol addresses the fact that with every minute that passes without CPR being performed, the likelihood of survival decreases by 7-10\% (2011).

Quality chest compressions can also affect patient outcome when ALS arrives to administer a shock with an AED, which is used to convert an irregular heart rhythm to a regular one. According to Cobb et al. (1999) performing 90 seconds of CPR prior to the use of an AED was associated with increased patient survival when Emergency Medical Services (EMS) response intervals were 4 minutes or longer. Supplying adequate perfusion to the cardiac muscle of the heart allows the heart to respond to a shock by an AED more favorably than if oxygenated blood was lacking.

With the majority of sudden cardiac arrests occurring at home, an undetermined arrival time of emergency personnel, and ALS being affected by levels of perfusion, it is necessary to better understand the effects of fatigue on the quality of compressions in the layperson rescuer as well as the professional rescuer.

\section{Statement of the Problem}

The increased frequency of chest compressions in the current BLS protocol, $\geq 100$ per minute, while more likely to result in survival of the victim, is also likely to result in earlier fatigue among rescuers. The effects of physical fitness on performance of chest 
compressions have not been adequately studied in laypersons and professional rescuers. The rate of fatigue may affect the duration that single-rescuer CPR can be performed, and this has implications for ALS response times necessary to provide continuity of care between the first responder and professional rescuers.

\section{$\underline{\text { Statement of the Purpose }}$}

The purpose of this study was to investigate how physical fitness, compression rate $(\geq 80 \mathrm{cpm}$ versus $\geq 100 \mathrm{cpm})$, gender, and lay rescuer versus professional rescuer status, affected time to fatigue during performance of continuous chest compressions.

\section{$\underline{\text { Delimitations }}$}

1. Study subjects included professional and layperson rescuers.

2. This study involved a physical fitness evaluation, using the YMCA 3-minute step test and bench press test, prior to participation.

3. This study evaluated the rate of compressions, time to fatigue, heart rate, and RPE.

4. This study included subjects from San Luis Obispo County.

5. This study occurred between the months of January and August, 2014.

\section{$\underline{\text { Limitations }}$}

1. Compression performance on the manikin might be different from an actual arrest due to the rise in catecholamine concentrations in the rescuer and other unforeseen variables.

2. This study was a simulated resuscitation and did not represent the environment faced by many professional rescuers.

3. The trials of compressions performed back-to-back might have produced a learning or fatigue effect. 
4. Certain information such as goal of study and not being blinded to the ratio being performed might have biased the results.

5. Some of the ECG data were lost due to corruption of the storage disk.

6. The age of the subject might be a factor in their physical performance.

\section{$\underline{\text { Hypotheses }}$}

1. The mean heart rate will be greater at $\geq 100$ compressions per minute (cpm) than at $\geq 80 \mathrm{cpm}$

2. The mean Rating of Perceived Exertion will be greater at $\geq 100 \mathrm{cpm}$ than at $\geq 80$ cpm.

3. The mean duration of compressions will be greater at $\geq 80 \mathrm{cpm}$ than at $\geq 100 \mathrm{cpm}$.

4. Performing $\geq 80 \mathrm{cpm}$ first will increase compression duration during the second trial.

5. Performing $\geq 100 \mathrm{cpm}$ first will increase heart rate during both trials.

6. Rating of Perceived Exertion will be greater during both trials when performing $\geq 100$ cpm first.

7. Professional rescuers will have a greater endurance than layperson rescuers.

8. Professional rescuers will have a lower Rating of Perceived Exertion than layperson rescuers.

9. Professional rescuers will have a lower mean heart rate than layperson rescuers.

10. Men will have a greater endurance than women.

11. Men will have a lower Rating of Perceived Exertion than women.

12. Men will have a lower mean heart rate than women.

13. A high YMCA Bench Press score will result in a longer duration of compressions.

14. A lower YMCA Step Test score will result in a longer duration of compressions. 


\section{Definition of Terms}

1. Advanced Life Support (ALS):

A higher level of emergency medical care, usually provided by EMT-intermediates or paramedics. Typically ALS includes invasive techniques such as IV therapy, intubation, and/or drug administration (Mosby's, 2009).

2. Asystole:

A life-threatening cardiac condition characterized by the absence of electrical and mechanical activity in the heart. Clinical signs include apnea and lack of pulse. Without cardiac monitoring, asystole cannot be distinguished from ventricular fibrillation (Mosby's, 2009).

3. Automatic External Defibrillator (AED):

A portable device that checks the heart rhythm. If needed, it can send an electric shock to the heart to try to restore a normal rhythm (National Institutes of Health, 2013).

4. Cardiac output:

The volume of blood ejected from the left side of the heart in one minute (MerriamWebster, 2013).

5. Cardiopulmonary Resuscitation (CPR):

A procedure designed to restore normal cardiac activity and breathing after cardiac arrest that includes the clearance of air passages to the lungs, the mouth-to-mouth method of artificial respiration, and heart massage by the exertion of pressure on the chest (Merriam-Webster, 2013). 
6. Chain of Survival:

Refers to a series of actions that, when put into motion, reduce the mortality associated with cardiac arrest. Like any chain, the chain of survival is only as strong as its weakest link. The four interdependent links in the chain of survival are: early access, early CPR, early defibrillation, and early advanced cardiac life support (American Heart Association, 2013).

7. Duty cycle:

The percentage of one period in which the heart is in the contraction phase. A period is the time it takes for the heart to complete a contraction-to-rest cycle.

8. Fatigue:

Muscular weakness brought on by physical exertion in performing an extended bout of compressions.

9. Hemodynamics:

A branch of physiology that deals with the circulation of the blood (MerriamWebster, 2013).

10. Hypovolemia:

Decrease in the volume of the circulating blood (Merriam-Webster, 2013).

11. Layperson rescuer:

A layperson who only has a moral or ethical obligation to provide care and had basic life support (BLS) training, but no formal training in advanced life support procedures.

12. Leaning:

When a rescuer deviates from a vertical arm position. 
13. Monitor-defibrillator:

An emergency device that includes both a monitor to display the electrocardiogram and a direct current cardioverter/defibrillator.

14. Perfusion:

To force a fluid through (an organ or tissue) especially by way of the blood vessels (Merriam-Webster, 2013).

15. Professional rescuer:

Emergency technician who has a job-defined duty to provide care and has been trained in more advanced life support procedures beyond BLS.

16. Pulseless Ventricular Tachycardia:

Is a tachycardia, or fast heart rhythm, that originates in one of the ventricles of the heart and is characterized by greatly diminished or lack of cardiac output.

17. Randomized crossover study:

Subjects are assigned to receive all the different treatments or exposures in a study in a random order.

18. Residual Volume (RV):

The amount of air remaining in the lungs at the end of a maximum expiration (Mosby’s, 2009).

19. Sudden cardiac arrest:

A condition in which the heart suddenly and unexpectedly stops beating. If this happens, blood stops flowing to the brain and other vital organs (National Institutes of Health, 2013). 
20. Ventricular Fibrillation:

A heart rhythm in which uncoordinated myocardial depolarizations and contractions results in inadequate cardiac output to sustain life. 
Chapter 2

\section{REVIEW OF LITERATURE}

\section{$\underline{\text { Method }}$}

This review of literature resourced articles from the following databases: MEDLINE, SPORTDiscus, CINHAL, PubMED, Web of Knowledge (ISI), ERIC (EBSCO), Science Direct, and PsycINFO (EBSCO). The following key words were used to find the research articles: Cardiopulmonary Resuscitation (CPR), continuous compressions, compression-to-ventilation ratios, compression rates, rescuer, and fatigue. Other peer-reviewed research articles were found using the keywords Emergency Medical Services (EMS), response time, cardiac perfusion, and patient survival. Out of the articles found within Biology, Kinesiology, and Education databases, there were 34 research articles pertaining to the desired inclusion criteria.

\section{Discussion}

This literature review examines how different compression rates and ratios affect rescuer fatigue and the quality of compressions during CPR. The focus was on a search of literature pertaining to the effect of continuous compressions and compression rates on rescuer fatigue, arterial perfusion, and survival of the patient. There are no single studies that show a difference between layperson and professional rescuers and the lack of research further highlights the need for effective data collection in this area. This review also examines the response times of emergency personnel and how standard response intervals compare to actual response times that have occurred in the field. This research examines to what extent rescuer fatigue plays a part in patient survival. The review of 
literature for all these categories will help to bring into focus important issues effecting fatigue and highlight needed changes in the present emergency response system.

The compression rate at which CPR is performed can have a detrimental effect on the rescuer depending on how long the rescuer may need to continue compressions. According to the AHA in 2011, guidelines for cardiopulmonary resuscitation recommend a chest compression rate of at least 100 compressions per minute (cpm). The 2010 European Resuscitation Council (ERC) CPR guidelines recommend at least $120 \mathrm{cpm}$ (Idris et al., 2012). A study by Idris et al. (2012) examined compression rates during outof-hospital $(\mathrm{OOH}) \mathrm{CPR}$ to determine whether there is a relationship with patient outcome. The study included patients aged $\geq 20$ years who had suffered an $\mathrm{OOH}$ cardiac arrest. Data were abstracted from monitor-defibrillator recordings. Between December, 2005, and May, 2007, 3098 patients were included in the study. The mean age of subjects was $67 \pm 16$ years and the mean compression rate was $112 \pm 19$ /minute. The return of spontaneous circulation (ROSC) rates peaked at a compression rate of $\approx 125 /$ minute and then declined. According to this study, both the AHA and ERC would benefit from raising their standard for $\mathrm{cpm}$ at least where the ROSC is concerned. The survival to hospital discharge was not associated with the $\approx 125 /$ minute rate.

An observational study by Monsieurs et al. (2012) investigated the relationship between chest compression rate and compression depth. Researchers hypothesized that with a faster compression rate they will see an associated decrease in depth. Researchers evaluated chest compressions for 133 healthcare professionals. The depth $(\geq 0.5 \mathrm{~cm}=$ clinically significant) of compression was compared for rates $<80 \mathrm{cpm}, 80-120 \mathrm{cpm}$, and $>120 \mathrm{cpm}$. The majority of subjects $(62 \%)$ were between $80-120 \mathrm{cpm}$ at a depth of 
$4-5 \mathrm{~cm}$. For a compression rate of $80-120 \mathrm{cpm}$ the average depth was $4.5 \mathrm{~cm}$. There tended to be a lower compression depth as the rates increased. Rates $>145 \mathrm{cpm}$ resulted in a depth of $<4.0 \mathrm{~cm}$. Researchers suggest avoiding excessive compression rates due to the association between higher compression rate and lower compression depth. This study, in relation to the current study, shows a correlation between higher compression rates and rescuer fatigue depicted by a decrease in compression depth.

A study by Abella et al. (2005) looked at how in-hospital chest compression rates compared to published international guidelines. Researchers observed adult cardiac arrests at three different hospitals between April, 2002, and October, 2003. Out of 97 cardiac arrests, which included 813 minutes of chest compressions, $36.9 \%$ were $<80$ cpm. Survivors or those with initial ROSC had mean chest compression rates of $90 \pm 17$ $(\mathrm{p}=0.003)$. Researchers concluded that in-hospital chest compression rates were below published resuscitation recommendations, and suboptimal compression rates correlated with poor ROSC.

A comparative study by Feneley et al. (1988) investigated initial resuscitation success and 24-hour survival in dogs after 30 minutes of manual CPR at rates of 60/minute and 120/minute. In this article researchers mentioned a conflict between studies by Maier et al. (1986) and Wolfe et al. (1988) and Kern et al. (1986) on whether the higher compression rate (120/minute) is the optimal compression rate for survival from cardiac arrest. Researchers wanted to resolve the issue of whether faster manual CPR compression rates have the potential to improve survival. When researchers considered the entire 24 hour period of observation after 30 minutes of CPR, survival was significantly greater at a manual compression rate of 120 /minute than at a compression 
rate of $60 /$ minute $(p<0.005)$. Researchers determined that survival is probably related to greater aortic diastolic and coronary perfusion pressures attained with high-rate compressions.

The article by Maier et al. (1986) evaluated 5 years of investigation into hemodynamic support during CPR. Researchers used high-impulse CPR (120 compressions per minute) on dogs to determine whether systemic and coronary blood flow were optimized. They found that manual chest compressions of high velocity, moderate force, and brief duration at a rate of 120/minute improved blood flow. At the time of the study, researchers suggested that the AHA guidelines be changed to recommend a manual chest compression rate of 120/minute during closed-chest cardiac massage.

In the study by Wolfe et al. (1988) researchers measured coronary blood flow in dogs to determine an optimal chest compression rate. High-impulse compressions were performed on a dog in the supine position at a compression rate of between 60150/minute. Diastolic perfusion time decreased linearly with compression rate and limited coronary perfusion at rates $>120$ /minute. Coronary blood flow was determined by diastolic aortic pressure and perfusion time. A compression rate of 120/minute did optimize coronary blood flow.

The study by Kern et al. (1986) looked at three different methods of compressions and the 24 hour survival of 30 dogs. Researchers evaluated high-impulse compressions at a rate of $120 \mathrm{cpm}(10: 1 \mathrm{C}: \mathrm{V}$ ratio), interposed abdominal compressions at a rate of 60 cpm (50\% duty cycle), and standard CPR at $60 \mathrm{cpm}$ (50\% duty cycle, 5:1 C:V ratio). Researchers used end-tidal carbon dioxide to determine the efficacy of CPR. 
Compressions continued until defibrillation at minute twenty. The two techniques were reported to produce higher cardiac output, aortic pressures or coronary perfusion pressures than standard CPR. According to the previous studies by Maier et al. (1986) and Wolfe et al. (1988) a high-impulse compression rate of $120 \mathrm{cpm}$ optimized perfusion pressure and blood flow. The current study by Kern et al. (1986) found that highimpulse, interposed abdominal, and standard compressions were all effective in initial resuscitation. Researchers suggested three possible explanations that might have led to this discrepancy: The experimental design between studies, the quality of the standard CPR being compared to the other methods, and to a possible difference in anesthetic agents.

A study by Field, Soar, Davies, Akhtar, and Perkins (2011) evaluated the effect of different compression rates on quality variables (compression depth, duty-cycle, leaning, and performance decay over time). Researchers had 20 healthcare professionals perform 2 minutes of continuous chest compressions at rates of 80,100,120, 140 and 160/minute. An instrumented manikin was used and trials were random. It was determined that rates above 120/minute had the greatest impact on reducing chest compression quality. Chest compression quality in terms of depth, duty-cycle, leaning, and decay in performance were adequately maintained at a rate of 100-120/minute for a period of 2 minutes.

The physical fitness of the rescuer can play an important part in reducing rescuer fatigue. Having an adequate level of muscular and aerobic endurance may allow the rescuer to maintain the quality of chest compressions and have a positive impact on the patient's outcome. In a study by Ock, Min Kim, Chung, and Hong Kim (2011) researchers evaluated the influence of physical fitness on the performance of continuous 
chest compressions for 5 minutes. Researchers also looked at the effect of the rescuer's weight, height, and gender on the quality of compressions. Before CPR was performed researchers assessed the 47 participants' physical fitness by measuring muscular strength, power, endurance, reactive agility, and maximum aerobic capacity. After the first minute, there was a significant reduction in correct compressions. At the first minute the percentage of correct compressions was 78.8\% and declined each minute after from $57.2 \%$ in the second, $43.4 \%$ in the third, $36.5 \%$ in the fourth, and $28 \%$ in the fifth. Muscle strength had a direct influence on the quality of chest compressions. The results suggested that CPR providers should consider a muscle-strengthening program in order to improve the survival rate from cardiac arrest.

An article by Hansen et al. (2012) discussed the effects of physical fitness on the quality of compressions in Cardiocerebral Resuscitation (CCR) (i.e., no ventilations). The researchers hypothesized that a high cardiopulmonary exercise capacity and/or muscle strength can delay the development of fatigue and preserve the quality of compressions. The study evaluated 15 healthcare professionals, between the ages of 2152, while they performed 15 minutes of CCR. Cardiopulmonary exercise tests, and hand grip strength tests were performed to acquire a baseline physical assessment. Depth and frequency of compressions were monitored. Blood lactate concentrations and heart rate were assessed. Ventilatory threshold was related to the quality of compression within the first 5 minutes $(\mathrm{p}<0.05)$. Maximal elbow extension strength was related to quality after 5 minutes $(\mathrm{p}<0.05)$. It was concluded that ventilatory threshold is significantly related to CCR quality and those healthcare professionals regularly participating in CCR should maintain a high level of aerobic exercise capacity. 
A study by Russo et al. (2011) examined the impact that physical fitness and biometric data, such as gender, body mass index (BMI), and heart rate (HR), have on the quality of external chest compressions (ECC). Researchers evaluated 40 (30 male and 10 female) healthcare professionals during two 9-minute trials of ECC on a manikin using two different compression-to-ventilation ratios (30:2 and 15:2). Contrary to the current study, researchers used cycle and rowing ergometry to assess physical fitness prior to testing. Subjects with a lower BMI and better physical fitness performed better with less fatigue. Heart rate during rowing was a better predictor of ECC quality than lower body exercise performance on the cycle ergometer. These researchers strongly suggested switching rescuers every 2 minutes to alleviate fatigue.

According to Kern et al. (2002) increasing compressions leads to higher vascular pressure resulting in a more productive coronary perfusion rate supplying the vital organs necessary to sustain life. These researchers investigated the effects of a $15: 2 \mathrm{C}: \mathrm{V}$ ratio versus continuous chest compressions on hemodynamic (circulation of blood) compromise in 12 minutes of CPR in swine. This study determined that the average time from stopping to starting compressions to delivering ventilations was 16 seconds. This interruption resulted in no circulatory support for $60 \%$ of the resuscitation time. Continuous chest compressions resulted in not only an increase in the number of compressions per minute, but also improved arterial pressure, possibly affecting patient outcome from cardiac arrest.

Another study by Kern et al. (1992) examined chest compression rates during CPR in humans. The prospective, cross-over trial compared rates of 80 and $120 \mathrm{cpm}$ using end-tidal carbon dioxide as an indicator of efficacy. Carbon dioxide levels 
increased with the increased compression rate. This finding indicated that with the higher rate of compressions per minute there was improved blood flow measured by the gas exchange in the pulmonary system. This correlation also showed a relationship between the rapid compression rate of $120 \mathrm{cpm}$ and patient survival. Out of the 23 patients who had ROSC, and were successfully resuscitated, the mean end-tidal carbon dioxide partial pressure was $17.9 \pm 2.2 \mathrm{~mm} \mathrm{Hg}$ during the 2 minutes of testing. Patients who could not be resuscitated had a mean level of $10.4 \pm 1.0 \mathrm{~mm} \mathrm{Hg}$. Researchers also concluded that using a metronome for rate guidance during compressions had a positive impact on exhaled end-tidal carbon dioxide levels and may improve patient outcome from cardiac arrest. Both of the Kern et al. studies showed a positive improvement in perfusion with an increase in compression rate, yet neither of the studies touched on how rescuer fatigue might affect the quality of compressions while utilizing a higher compression rate.

A randomized cross-over study by Trowbridge et al. (2009) examined a comparison between the quality of CPR among women performing hands-only CPR (i.e., compressions only), and a rate of 30:2 C:V CPR. Researchers evaluated the rate and depth of compressions, with hands-only CPR, for a 10-minute interval. Fatigue (compression force, rate of perceived exertion, and blood lactate concentrations) and biomechanical changes were evaluated with each type of CPR. These statistics were evaluated using a mixed model, repeated measures, cross-over design, as well as changes in electromyography and joint kinetics and kinematics. Only 17 of the 20 participants in the study were able to complete 10 minutes of hands-only CPR. All 20 participants, performing 30:2 CPR, completed the full 10 minutes. Force and depth of compressions 
were lower in the hands-only CPR compared to the 30:2 CPR and both declined within the first 2 minutes.

With the increasing compression rate we see a noticeable increase in rescuer fatigue and, consequently, a decrease in the quality of chest compressions. Following the increasing compression protocol a number of studies noticed a sharp decline in performance within two minutes of the onset of testing and rescuers were unable to determine when their fatigue started to affect the quality of compressions. In a study by McDonald et al. (2013) the effect of rescuer fatigue on Cardiopulmonary (CPR) performance was investigated in accordance with the European Resuscitation Council (ERC) guidelines. Researchers evaluated 65 health science students as they performed 5 minutes of CPR following ERC guidelines. Subjects were asked to self-evaluate at what point fatigue managed to have an effect on the quality of their compressions. Almost half of the decline in the depth of chest compressions occurred between the first and second minutes of CPR. These researchers concluded that rescuer fatigue affects compression quality within the first 2 minutes of CPR and rescuers are not a reliable source for determining their own fatigue.

A study by Hightower, Thomas, Stone, Dunn, and March (1995) evaluated the decay in the quality of compressions over time. The researchers studied nursing assistants who had regular experiences with Cardiopulmonary Resuscitation (CPR) performed in the emergency room. Each of the 11 subjects performed 5 minutes of closed-chest compressions and they were asked to verbally announce when they felt the quality of their compressions was not effective due to fatigue. Researchers analyzed the number of compressions per minute and the adequacy of those compressions. Even 
though the rate of compressions did not decline over time, the quality of compressions decreased linearly over the 5-minute period. Subjects were unable to recognize at what point, during the 5-minute period, fatigue affected their ability to perform adequate compressions.

Even though these studies show that fatigue was affecting the rescuer's performance of quality compressions, one study showed better compressions and quality during continuous chest compressions. A study by Heidenreich, Berg, Higdon, Ewy, and Kern (2006) compared standard CPR to continuous chest compression CPR and rescuer fatigue. This prospective, randomized crossover study incorporated 53 medical students who participated in 9 minutes of CPR per method with a rest period of two days between methods. Students were evaluated primarily on the number of adequate chest compressions with a minimum depth of $38 \mathrm{~mm}$. Even though the rate of decline of adequate compressions per minute was greater during continuous chest compressions compared to standard CPR, there were significantly more adequate compressions during continuous chest compression versus standard CPR during the first two minutes.

Research looking into the effects of continuous compression and rescuer performance evaluated the differences in fatigue and the quality of compressions between men and women. A study by Ashton et al. (2002) determined the effects of rescuer fatigue while performing chest compressions within a 3 minute period. Participants were doctors and nurses who worked in the intensive care unit of a general hospital in the United Kingdom. Rate and quality of compressions were measured during two sessions of 3 minutes each separated by a 30 second rest. Out of the forty subjects tested, 20 men and 20 women, there was a significant positive correlation between the height and weight 
of participants and the number of quality compressions. Female participants administered fewer quality compressions than their male counterparts. Rescuer fatigue was determined to affect the quality of chest compressions over a 3 minute period. It was recommended to lessen the interval to under 3 minutes for rotating the rescuer performing compressions.

Other research investigated whether gender, age, height, weight, and professional status would affect the quality of chest compression in CPR. Research by Ochoa, Ramalle-Gomara, Lisa, and Saralegui (1998) investigated whether a rescuer's gender, age, weight, height or professional status would influence a reduction in the quality of chest compressions attributed to fatigue. This study used participants employed within an intensive care and emergency department who were trained in Cardiopulmonary Resuscitation (CPR) and ranged in age from 25 to 45 years. A total of 38 participants took part in the study of performing 5 minutes of continuous chest compressions. After a statistical analysis, using analysis of variance and three models of multiple linear regression, it was determined that a decrease in the quality of compressions occurred after the first minute of CPR. This effect on quality was not dependent on gender, age, height, weight, or rescuer profession. The researchers also concluded that the person performing compressions did not adequately perceive the decline in compression quality.

A study conducted by Riera, Gonzalez, Alvarez, Fernandez, and Saura (2006) reviewed qualitative and quantitative data from 23 participants performing two minutes of uninterrupted compressions among patients in cardiac arrest. The participants were members of a hospital intensive care unit cardiac arrest team. Pulse oximetry was used to measure oxygen saturation and heart rate after performing compressions for 1 minute and 
2 minutes. Fatigue was measured using a visual analogical scale. These investigators concluded that health professionals trained in CPR were able to tolerate uninterrupted chest compressions for 2 minutes without jeopardizing the quality of compressions.

A review article by Cunningham, Mattu, O’Connor, and Brady (2012) compared continuous chest compressions to conventional CPR and examined possible ways of reducing interruptions during compressions. Researchers discussed sources of chest compression interruption such as: pulse determination, cardiac rhythm interpretation, electrical defibrillation, airway management, and vascular access. Cunningham et al. (2012) stressed the need to reduce the time of interruptions thus allowing for increased perfusion pressure and improved patient outcome from cardiac arrest.

The previous review of literature describes understandable correlations between rescuer fatigue and an increase in either the frequency or ratio of chest compressions to ventilations. A majority of articles on compression rates suggest that rescuers should maintain at least $120 \mathrm{cpm}$ in order to allow for proper compression depth and at the same time creating optimal perfusion rates for improved patient outcome. Compression rates $>120 \mathrm{cpm}$ produced noticeable fatigue as indicated by a decrease in compression depth. The research states that physical fitness and muscular and aerobic endurance play a major part in patient survival, and may be the difference between life and death if a single rescuer must maintain perfusion of the patient until ALS arrives. As the ratio of compressions-to-ventilations increases, we notice an improvement in the number of compressions leading to a beneficial arterial perfusion rate and improved patient outcome, yet we also notice the detrimental effect this increase has on a rescuer's level of fatigue and consequently the quality of compressions administered. It is important to 
recognize that in studies such as those conducted by Hightower et al. (1995) and McDonald et al. (2013) subjects were unable to determine at what point fatigue had compromised the quality of their compressions. The data from studies such as McDonald et al. (2013) would suggest switching rescuers every 2 minutes to avoid fatigue. Table 1 shows the different ratios and rates that each study examined along with the duration of the trial and the research findings.

The following review of literature focuses on evaluating Emergency Medical Services (EMS) response times to an out-of-hospital, life-threatening cardiac event. The review of response times takes into account what is considered a valid response time and which periods of the arrest are the most vital to survival. This critical time between the emergency call and the arrival of Advanced Cardiac Life Support (ACLS) might determine the outcome of the patient. The delayed arrival of Advanced Life Support (ALS) may hinder the survival of the patient due to fatigue of the rescuer performing Cardiopulmonary Resuscitation (CPR). Physical fatigue can occur in a situation in which a single rescuer performing CPR must continue without a second rescuer providing additional assistance. The following studies examine how many minutes on average a rescuer should be prepared to administer CPR before additional assistance presents itself and how this interval may affect patient outcome.

According to a study by Narad and Driesbock (1999) criteria for establishing standard response intervals have been implemented in 33 out of 58 counties in California. The study suggested that these standards were only recommendations and further compliance by these EMS systems should be addressed. The standards included the improvement of the system in place by lowering the cost of care to improve quality and 
Table 1. A literature review summary of compression rates, ratios, and findings. The chart headings show the three different compression-to-ventilation ratios $(15: 2,30: 2$, 50:2) and continuous compressions (CC). The heading ( $\mathrm{min}$ ) is the duration of the compression trial. The heading (rate) refers to the number of compression per minute (cpm).

\begin{tabular}{|c|c|c|c|c|c|c|c|c|}
\hline Researcher & Year & $15: 2$ & $30: 2$ & $50: 2$ & $\mathrm{CC}$ & Min & Rate (cpm) & Findings \\
\hline Idris et al. & 2012 & & & & $x$ & 5 & 125 & $\begin{array}{l}\text { Return of spontaneous } \\
\text { circulation peaked at } 125 \mathrm{cpm} \\
\text { and then declined }\end{array}$ \\
\hline Monsieurs et al. & 2012 & & & & $x$ & & $80-120$ & $\begin{array}{l}\text { Higher comp. rate resulted on } \\
\text { lower comp. depth. }\end{array}$ \\
\hline Feneley et al. & 1988 & & & & $x$ & 30 & 60,120 & $\begin{array}{l}\text { Higher compression rate } \\
\text { resulted in better perfusion } \\
\text { pressures }\end{array}$ \\
\hline Maier et al. & 1986 & & & & $\mathrm{X}$ & & 120 & $\begin{array}{l}\text { Optimized systemic and } \\
\text { coronary blood flow }\end{array}$ \\
\hline Wolfe et al. & 1988 & & & & $\mathrm{X}$ & & $60-150$ & $\begin{array}{l}\text { Comp. rate of } 120 / \mathrm{min} . \\
\text { optimized coronary blood flow. }\end{array}$ \\
\hline Field et al. & 2011 & & & & $x$ & 2 & $\begin{array}{l}80,100,120 \\
140,160\end{array}$ & $\begin{array}{l}\text { Rate of } 100-120 \mathrm{cpm} \text { for } 2 \mathrm{~min} . \\
\text { maintained quality variables. }\end{array}$ \\
\hline $\begin{array}{l}\text { Ock } \\
\text { et al. }\end{array}$ & 2011 & & & & $x$ & 5 & & $\begin{array}{l}\text { Muscle strength had direct } \\
\text { influence on quality of comp. }\end{array}$ \\
\hline $\begin{array}{l}\text { Hansen } \\
\text { et al. }\end{array}$ & 2011 & & & & $x$ & 15 & & $\begin{array}{l}\text { High level of aerobic exercise } \\
\text { capacity is recommended due } \\
\text { to relation to CCR quality }\end{array}$ \\
\hline Russo et al. & 2011 & $x$ & $x$ & & & 10 & & $\begin{array}{l}\text { Upper body }=\text { better predictor } \\
\text { of ECC. Switch rescuers every } 2 \\
\text { min. to alleviate fatigue. }\end{array}$ \\
\hline $\begin{array}{l}\text { Kern } \\
\text { et al. }\end{array}$ & 2002 & $x$ & & & $x$ & 12 & & $\begin{array}{l}\text { Continuous chest compressions } \\
\text { increased number of comp. per } \\
\text { minute and improved arterial } \\
\text { pressure }\end{array}$ \\
\hline $\begin{array}{l}\text { Trowbridge et } \\
\text { al. }\end{array}$ & 2009 & & $x$ & & $x$ & 10 & & $\begin{array}{l}\text { Force and depth decreased in } \\
\text { continuous versus } 30 \text { to } 2 \text { / } \\
\text { both declined w/in } 2 \text { min. }\end{array}$ \\
\hline $\begin{array}{l}\text { McDonald } \\
\text { et al. }\end{array}$ & 2012 & & $x$ & & & 5 & & $\begin{array}{l}\text { Fatigue affects quality w/in first } \\
2 \text { min./ rescuers could not } \\
\text { determine when fatigue set in }\end{array}$ \\
\hline $\begin{array}{l}\text { Hightower } \\
\text { et al. }\end{array}$ & 1995 & & & & $x$ & 5 & & $\begin{array}{l}\text { Rate did not decline/quality } \\
\text { decreased over } 5 \text { min./rescuers } \\
\text { could not determine when } \\
\text { fatigue set in }\end{array}$ \\
\hline $\begin{array}{l}\text { Heidenreich et } \\
\text { al. }\end{array}$ & 2006 & & $x$ & & $x$ & 9 & & $\begin{array}{l}\text { More adequate comp. } \\
\text { w/continuous versus } 30 \text { to } 2 \\
\text { during first } 2 \text { min. }\end{array}$ \\
\hline $\begin{array}{l}\text { Ashton } \\
\text { et al. }\end{array}$ & 2002 & & & & $x$ & 3 & & $\begin{array}{l}\text { Rescuer fatigue affected quality } \\
\text { over } 3 \mathrm{~min} \text {. / rotate rescuers } \\
\text { under } 3 \mathrm{~min} \text {. }\end{array}$ \\
\hline $\begin{array}{l}\text { Ochoa } \\
\text { et al. }\end{array}$ & 1998 & & & & $x$ & 5 & & $\begin{array}{l}\text { Decrease in quality after } 1 \\
\text { min./rescuer did not perceive } \\
\text { fatigue }\end{array}$ \\
\hline $\begin{array}{l}\text { Riera } \\
\text { et al. }\end{array}$ & 2006 & & & & $x$ & 2 & & $\begin{array}{l}\text { Able to tolerate } 2 \text { min. } \\
\text { continuous chest comp. } \\
\text { without jeopardizing quality }\end{array}$ \\
\hline
\end{tabular}


patient outcome. Differences were found by identifying the mechanisms used, by counties in California, to regulate response times. Each county was in control of their own EMS regulation by designating a Local EMS Agency (LEMSA), which would be the EMS authority within that geographic area. In California, the recommendation for response times by EMS ambulance units are 8 minutes in urban areas and 20 minutes rural areas. In other remote areas EMS personnel are urged to get there as quickly as possible. This response time is defined as the interval from when the call is received to when EMS personnel arrive on scene. These standards are only recommendations and there is no enforcement by a state regulation. According to this study there is no assurance by many counties that their EMS services are complying with state recommended standards. A limitation of this study is that it examined response times from a single state. The comparison to statewide standards might highlight regulatory differences that could affect patient outcome.

In a more recent quantitative study by Wang et al. (2013) researchers investigated the national characteristics of out-of-hospital Emergency Medical Services (EMS) responses in the United States. Investigators obtained research data from the 2010 National Emergency Medical Services Information System (NEMSIS) for 29 states in the U.S. Researchers examined characteristics, number, and incidence of EMS responses as well as the patients receiving care. One of the characteristics of EMS services was the time interval of response. The characteristics of the EMS response variable included times for response, at scene, and transport. Location type, response mode, and incident outcome were also analyzed. With over 17 million EMS responses, based on information from 29 of 50 states in the U.S. each year, there was an average response time of 5 
minutes. This time is well within the range of care for conditions such as cardiac arrest or myocardial infarction, which are time-critical. With response of EMS in the U.S. being so frequent, these data emphasized an increasing demand for the services of emergency personnel. This study was limited by data only being collected by states that used NEMSIS. One of the states not represented in this research was California.

The objective of a study by Weiss, Fullerton, Oglesbee, Duerden, and Froman (2013) was to determine if EMS response times have an impact on patients with a specific medical emergency. This study was conducted over a 13 month time period between June, 2006, and July, 2007, and data were collected from a metropolitan area with a population of 800,000 . Researchers quantified motor vehicle crash injuries, penetrating trauma, difficulty breathing, and chest pain complaints. Response times were established from the time the call center received the call until the arrival at scene. Out of the 2,164 subjects who met the inclusion criteria, a total of $955(44 \%)$ of subjects complained of chest pain. Forty-two $(21 \%)$ of subjects with chest pain had a response time that exceeded 8 minutes and 5 subjects had a response time between 4-8 minutes. This retrospective cohort study found that EMS response times for ambulances were faster (4.5 \pm 3.9 minutes) for trauma complaints compared to medical complaints $(5.9 \pm$ 3.2 minutes $)$ and chest pain $(6.0 \pm 3.5$ minutes $)$, and that longer response times were not associated with worse outcomes.

An observational study by Blackwell and Kaufman (2002) assessed the effect that response times (RT) have on patient survival in an urban EMS system. Data collection occurred between March and August, 1998, and analysis included priority 1, lifethreatening emergencies and responses using ambulance lights and sirens. County RT 
requirements define a priority 1 , life-threatening emergency call to be under 10:59 minutes. A priority 2 non-life-threatening emergency RT should be less than 12:59 minutes. Researchers showed a correlation between RTs less than 5 minutes (mortality risk $0.51 \%$ ) and improved survival. Response times greater than 5 minutes showed the mortality risk going up to $1.58 \%$. A response time of less than 5 minutes was associated with improved survival. Researchers concluded that maintaining this RT and survival rate would meet with financial constraints making it very difficult to achieve.

A case-control retrospective study by Blackwell, Line, Willis, and Hicks (2009) hypothesized that the advanced life support (ALS) RT of $\geq 10$ minutes and 59 seconds would not affect patient outcome. Study data were obtained from EMS transport in a metropolitan county, with a population of 750,000, between the months of January and December, 2004. A comparison was made between patients that fell within the set RT standard and those who fell outside such time. At the 95\% confidence interval (CI), priority 1 patients who waited longer than $10: 59$ minutes experienced between a $6 \%$ increase and a 4\% decrease in mortality when compared to patients who waited 10:59 minutes or less for ALS response. This study concluded that there was no evidence of increased mortality with ALS RT exceeding 10:59 minutes.

A study by Blanchard et al. (2012) investigated whether an EMS response time of 8 minutes was associated with patient mortality. This retrospective cohort study of adults lasted one year and assessed patients with a life-threatening event. High priorityresponse subjects were evaluated between January and December of 2006. The clinical effectiveness was called into question among patients with a RT $\geq 8$ minutes, $7.1 \%$ died, compared with $6.4 \%$ for patients with a RT $\leq 7$ minutes 59 seconds. The definition of 
response time, identifying who should receive rapid EMS response, and who may benefit from rapid EMS response all became clinical limitations in this analysis. These researchers understood that rapid EMS response might be worthwhile to certain patients, but highlighted the challenges in developing a system to determine who should or should not receive a rapid response and optimum response time.

Researchers Salvucci, Kuehl, and Clawson (2004) examined the EMS system and the challenges that it faced in becoming an effective part of the emergency response system. The authors highlighted the fact that response time is not just the driving interval, but a host of components between when the medical emergency starts and when ALS is administered. These researchers concluded that a comprehensive EMS information system was needed to match responses to patient needs. By focusing on the parts of the response that make a difference, patient condition, treatment, and outcome, a more knowledgeable response can allow all aspects of the system to work effectively.

The objective of a retrospective cohort study by Pons et al. (2005) was to evaluate the response time (independent variable) of paramedics, in an urban setting and see how it affected patient survival (dependent variable). Emergency dispatch logs were retrieved from the Denver Paramedic Division Dispatch Center for the one year period between January and December of 1998. This study controlled for illness severity, and the researchers used an 8-minute response time guideline to measure EMS quality of care. This response time was not associated with improved survival, but a 4-minute response time did show survival benefit for patients with intermediate or high risk of mortality. These investigators highlighted the need to recognize the interval for medical response and how minimizing delay with all the steps was essential to maximizing patient survival. 
Another retrospective cohort study by Gold, Fahrenbruch, Rea, and Eisenberg (2010) examined patient survival rates per minute from the collapse of the patient until EMS arrival. This study was conducted over a 24-year period (January 1, 1985December 31, 2008) and reviewed over three thousand cases of out-of-hospital cardiac arrest with ventricular fibrillation (VF) in King County, Washington. Researchers included patients whose arrests: occurred before EMS arrived; were shown to have VF or pulseless ventricular tachycardia (VT); were witnessed by bystander; and were of cardiac origin. Researchers discovered a decline in survival that had a significant relationship between $(\mathrm{p}$-value $=0.010)$ change of time from collapse to EMS arrival. The change in survival over time showed a steep decline between minute 4 and minute 10 . The decline in survival was not at a constant rate due to physiological alterations occurring during arrest. The observed survival differed from the predicted absolute survival rates, from logistic regressions, due to variations in the rise and fall in percentage of survival.

A 22-month case series study by Valenzuela et al. (1993) compared EMS RT with collapse-to-intervention intervals to determine which would have a greater impact on patient survival from pre-hospital sudden cardiac arrest. This study consisted of 118 patients that were involved in out-of-hospital resuscitation efforts by Tucson, Arizona, firefighter paramedics between October 1, 1988, and July 31, 1990. The mean collapseto-CPR and collapse-to-defibrillation intervals were both shorter in survivors (3.5 minutes and 2.1 minutes, respectively). Patients who received bystander CPR had a better chance of survival. This study concluded that the collapse-to-critical intervention 
intervals had a greater impact on patient survival from out-of-hospital sudden cardiac death due to VF than EMS response intervals.

A study by Campbell, Gratton, Salomone, Lindholm, and Watson (1994) examined how the time interval from "vehicle at scene" to "patient access" (VSPA) affected the mandated response time intervals of $<9$ minutes (code 1 ) and $<13$ minutes (code 2). This prospective observational study found that adding in a call-to-patient access interval decreased RT compliance. There were 20,941 emergency calls during the study period between June, 1991, and November, 1991. Out of the 63 code 1 emergency life-threatening calls, $90.5 \%$ were system response-time-compliants using 9-1-1 call-toscene intervals. This percentage dropped to $63.5 \%$ compliant using 9-1-1 call-to-patient access intervals, showing the effect VSPA had on system response time compliance. If the VSPA interval is prolonged, due to response interval ending on scene rather than at patient, it would not be a good indicator of when patient care is actually being administered.

The objective of a comprehensive literature search by Nichol et al. (1996) was to evaluate patient survival after out-of-hospital cardiac arrest while taking into account: differences in response times, proportion of bystander cardiopulmonary resuscitation (CPR), and type and tier of EMS system. Inclusion criteria consisted of articles published between 1966 and 1992. Inadequate data made the evaluation of the effectiveness of different EMS systems difficult. As far as the meta-analysis of data was concerned, these researchers concluded that a decreased response time and the use of a two-tier EMS system (two types of providers and/or vehicles respond) may help significantly toward patient survival. 
A number of studies evaluated response times within EMS systems outside of the United States. A study by Do, Foo, Ng, and Ong (2013) aimed to analyze ambulance response times (ART), in Singapore, Malaysia, by using quantile regression with the standard ordinary least squares (OLS) model. Data were collected from the Cardiac Arrest and Resuscitation Epidemiology (CARE) prospective study, which had been collecting information on geographic location of prehospital cardiac arrest and ambulance calls in Singapore since 2001. Data from 31,896 emergency calls over a 5-month period between January and May of 2006 were analyzed. Researchers looked at factors contributing to longer ART such as gender and ethnicity versus call volumes within the last hour. Estimates showed that ART increased as a result of call volumes. Each additional call per hour increased ART by $93\left(90^{\text {th }}\right.$ percentile $)$ and $57\left(50^{\text {th }}\right.$ percentile $)$ seconds (respective of percentile of ART). Response time of the ambulance was measured in minutes from time between call center notification until arrival at scene. This study found that the volume of calls in the past hour was more significant in the system level than the patient level factors. Optimizing ambulance deployment was one area that could improve performance of ART in Singapore.

The objective of a study by Peleg and Pliskin (2004) was to monitor Israeli ambulances with geographic information systems (GIS) in order to model response times and support strategies for improved deployment of ambulances. This retrospective review evaluated two regional districts over a 12-month period between 1996 and 1997. The mean response times in Carmel and Lachish districts were 12.3 and 9.2 minutes, respectively. Within an 8 -minute standard response time, only $34 \%$ of calls were responded to on time in the Carmel district. In Lachish, $62 \%$ of calls were answered. 
This model showed that additional EMS posts should be established to help improve RT and patient survival.

Researchers De Maio, Stiell, Wells, and Spaite (2003) investigated whether survival, for at least $90 \%$ of cardiac arrest cases, as a function of time, would compare to the 8 minute or less standard EMS response interval. This 6 year study, based in Ontario, Canada, analyzed 9,273 patients who were treated for out-of-hospital cardiac arrest. Researchers concluded that the 8-minute interval was not supported by their data and suggested that the EMS defibrillation response interval for cardiac arrest be less than 8 minutes.

The previous literature review on EMS response times allows us a comprehensive investigation into how well the actual response times compare to the standard times mandated by each individual region. These data can give us a better understanding of how long a rescuer might have to perform CPR before ACLS arrives to take over compressions and extend further life saving measures. The majority of research makes the comparison between data they have collected to the EMS standard response interval of 8 minutes or less, for at least $90 \%$ of cardiac arrest calls. Some studies (Blackwell et al., 2002; Wang et al., 2013) revealed an association between response times of 5 minutes or less and improved survival. One study by Weiss et al. (2013) found that longer response times were not associated with worse outcomes. This particular study's main focus was more on trauma-related emergencies rather than medical and researchers evaluated emergency calls for chest pain and not an actual cardiac arrest. In the majority of cases a shorter response time was strongly associated with improved survival. Under these reviewed response times, rescuer fatigue would not be a factor as far as optimal 
care to the patient. Since EMS response times vary from call-to-call, the physical fitness of the rescuer might play a role in whether or not ALS is able to have a positive impact on patient survival. 


\section{Chapter 3}

\section{METHODS AND PROCEDURES}

\section{$\underline{\text { Restatement of the Purpose }}$}

The purpose of this study was to investigate how different compression rates affect rescuer fatigue during continuous chest compressions in the layperson versus the professional rescuer.

\section{$\underline{\text { Subjects }}$}

All Cal Poly faculty, staff, and student research with human subjects, as well as other research involving human subjects that is conducted at Cal Poly, must be reviewed by the Cal Poly Human Subjects Committee (HSC) for the protection of human subjects, the researchers, and the university. The Human Subjects Protocol form (Appendix A) was completed for approval by the committee. After HSC approval on January 22, 2014, subjects were recruited among the California Polytechnic State University, San Luis Obispo, campus population, the City of San Luis Obispo, and local Emergency Medical Services (EMS) in San Luis Obispo County, through the use of flyers, presentations, and email. Current CPR certification, or previous certification, was a prerequisite for participation in the study. This requirement expedited the trial procedure as it was not necessary to teach basic compression form and performance. Individuals were excluded from participating in the study if they were categorized as "High Risk" as established by the American College of Sports Medicine (ACSM, 2014).

This study sought participants from a wide range of age groups, both genders and all ethnic backgrounds. The prospective age range extended to 80 years due to the incidence of sudden cardiac arrest increasing with age (Mayo Foundation for Medical 
Education and Research, 2012); thus, senior adults are more likely to need to perform chest compressions on a spouse or peer. Persons with disabilities were not excluded in this study if they did not have exclusion criteria.

\section{$\underline{\text { Experimenters }}$}

Those who administered the study were all students/faculty in the Kinesiology Department at Cal Poly State University in San Luis Obispo, California. Christopher Cassidy and Dr. Davis were certified as Healthcare Providers in CPR through the American Heart Association (AHA) or the Red Cross. Christopher Cassidy, who had worked as a CPR Instructor through the AHA, and was a trained Emergency Medical Technician (EMT-B), was present to oversee compression technique and Basic Life Support (BLS).

\section{Chest Depth and Force Measurements}

In order to maintain continuity during testing, compression force needed to acquire the necessary depth of the chest $(38-51 \mathrm{~mm}$ or $1.5-2$ inches $)$ was monitored over the period of the study. This was done to see if the repeated pressure exerted on the chest of the manikin caused a wearing and made it easier to compress the chest over time. A test of the least amount of force, during a typical chest compression, was measured using a force platform with a Kistler DAQ system with a Bioware software program. A test was performed once a week for a total of six trials. Each trial consisted of 10 force measurements in Newtons $(\mathrm{FzN})$ for each of the two manikins (A, B). The mean force exerted over the 6 weeks of testing for manikin A was $514.50 \mathrm{FzN}$ and manikin B was 511.38 FzN. (Figure 1). Over the 6 weeks there was a low force measurement 


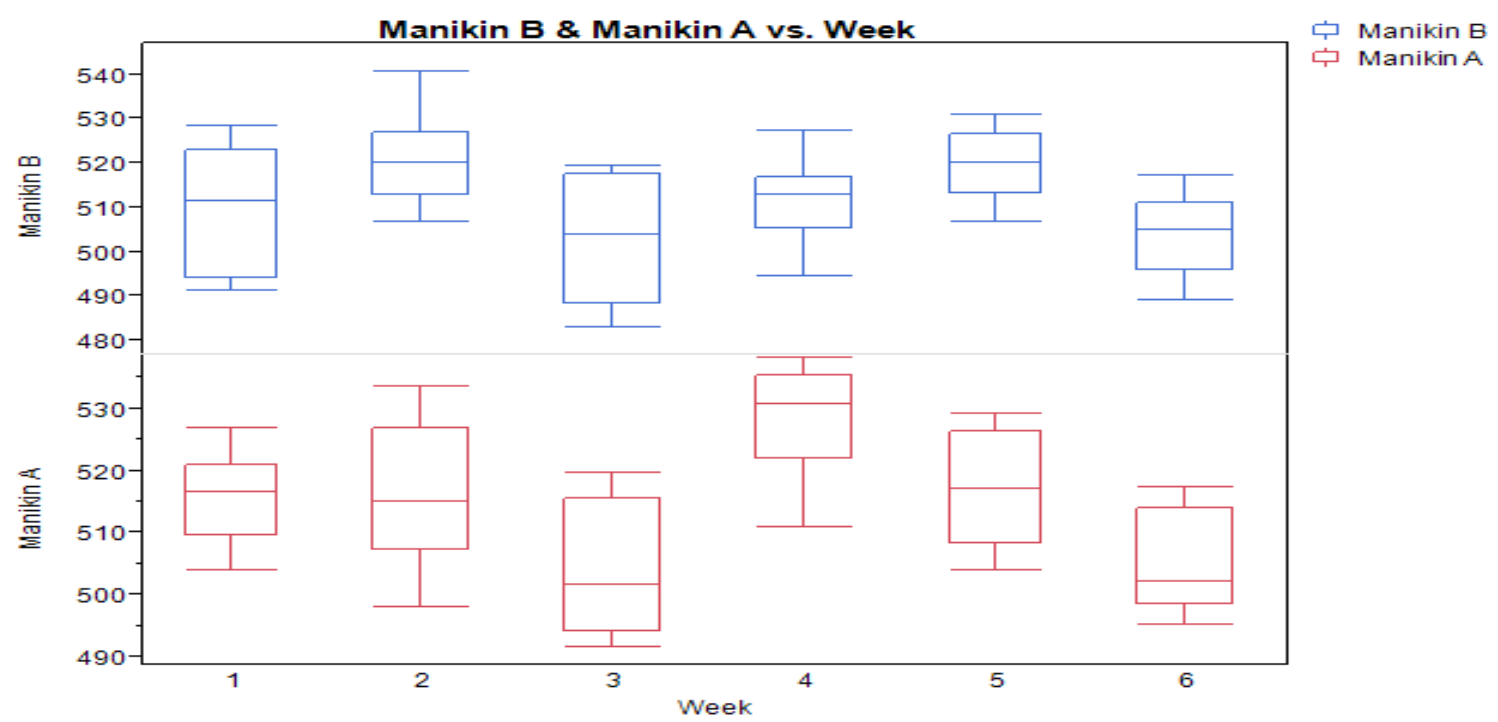

Figure 1. Differences in Forces Between Chest Compressions Performed on Both Manikins (A, B) Used in This Study. The line within the box indicates the mean compression force (Force on the $\mathrm{Z}$ Axis in Newtons, FzN) for each week for either manikin A or B. The lines extending from the top and bottom of each box is illustrating the standard error for each week for either manikin A or B.

of $504.13 \mathrm{FzN}$ and a high force value of $528.13 \mathrm{FzN}$, for manikin A. Manikin B had a low force measurement of $502.86 \mathrm{FzN}$ and a high force value of $520.67 \mathrm{FzN}$. For manikin A, there was a fluctuation in force between the 6 trials from a low of $-23.99 \mathrm{FzN}$ to a high of $12.37 \mathrm{FzN}$. Manikin B had a low force measurement between trials with a low of $-11.11 \mathrm{FzN}$ to a high of 17.81 FzN. Manikin A had a mean net compression force between 504.13 FzN and 528.13 FzN. Manikin B had a mean net compression force between 502.86 FzN and 520.67 FzN. The force needed to compress the chest of the manikin fluctuated over the six weeks of testing. Week six showed the least amount of force need to compress the chest of either manikin A or B compared to the preceding five weeks of testing. There was not a noticeable change, over the period of testing, in the force needed to compress the manikin's chest the proper depth, and this was not assessed to have an effect on data collection. 
The procedures utilized during testing sessions are diagrammed in Figure 2.

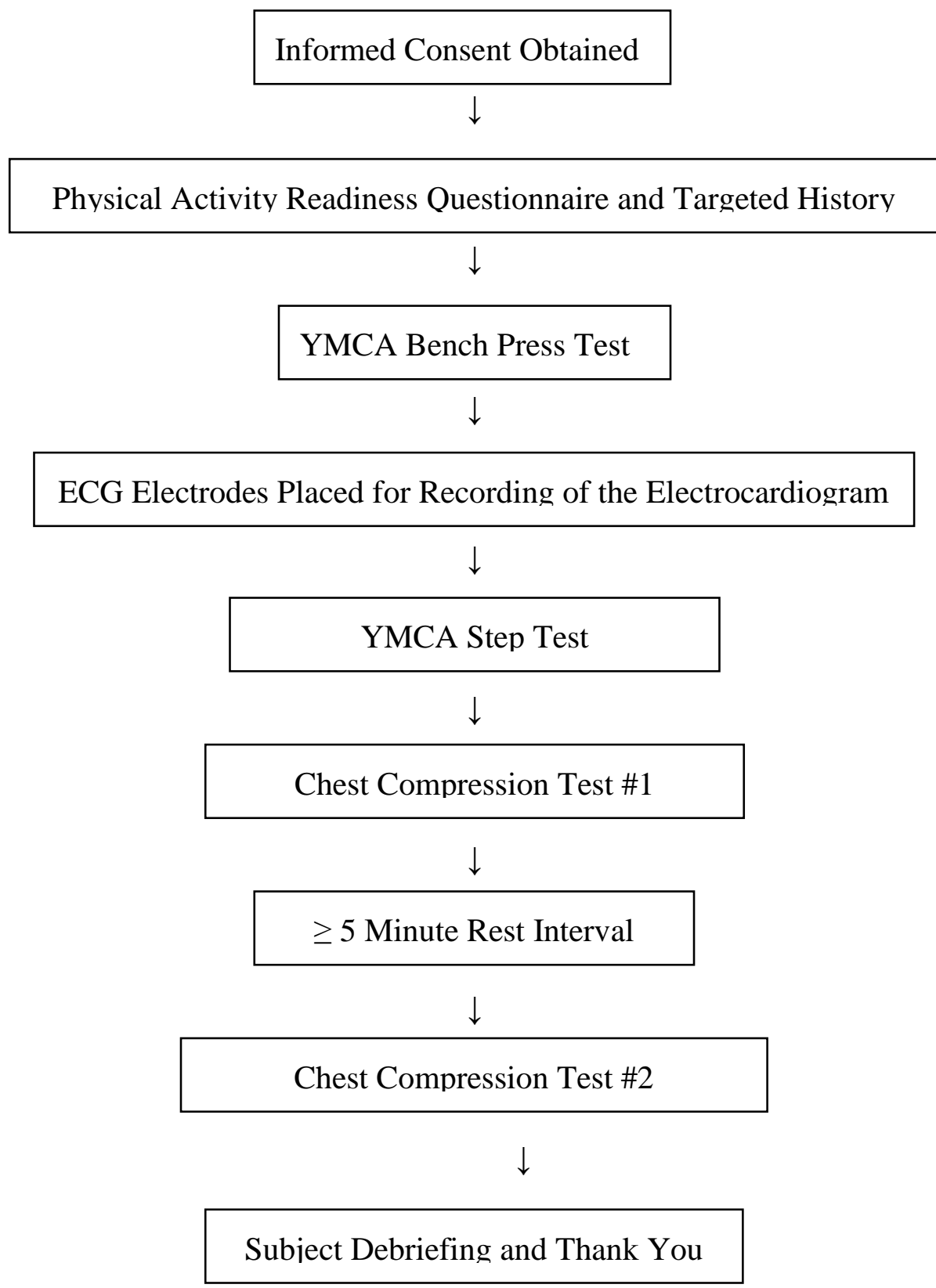

Figure 2. Testing Procedures 


\section{$\underline{\text { Informed Consent }}$}

Informed consent was obtained, and all subjects were instructed on the testing procedures and familiarized with what to expect before being asked to commit to the study. This study was for research purposes and all subject information and data collected were protected by confidentiality. At the initial meeting with the subject the experimenters had the subject complete an informed consent form (Appendix B) which described the study's purpose, procedures, risks, and steps to protect confidentiality.

\section{$\underline{\text { PAR-Q and Targeted History }}$}

All subjects participating in the study completed the Physical Activity Readiness Questionnaire (PAR-Q) (Appendix C) and were evaluated for past medical history. Experimenters asked the subject to answer the questions presented on the PAR-Q. Medical exclusion criteria, such as past history of heart disease or back pain, were contraindications for subject participation. If a subject answered "Yes" to any of the questions on the PAR-Q, they were excluded from participation in the study.

\section{$\underline{\text { YMCA Bench Press Test }}$}

Once the subject completed the necessary paperwork to move forward as a participant, the testing of the subject's physical fitness was conducted. Discontinuation of food intake was recommended at least one hour prior to physical fitness testing. Study subjects were asked to avoid food or drink containing stimulants that might enhance trial performance (e.g., caffeine). Physical activity was not recommended at least 1 hour prior to testing.

The first test to be administered was the YMCA bench press test (Appendix D) which gave experimenters a baseline measurement of the subject's muscular strength and 
endurance. Due to the increased possibility of injury using a maximal effort technique in untrained individuals, various prediction methods using sub-maximal loads have been suggested. The YMCA test was adopted in an attempt to evaluate chest strength and muscular endurance using a relatively light load. This test measured the number of continuous lifts of a weighted barbell (men: $80 \mathrm{lbs}$., women: $35 \mathrm{lbs}$.) the subject could perform during a single attempt. Once the subject could no longer keep pace with the metronome at a frequency of 60 beats per minute (30 lifts per minute), experimenters stopped the test and recorded the successful number of repetitions. The performed repetitions were compared to numbers, from a reference chart, within the subject's age bracket. This procedure determined whether the subject was categorized as having either "very poor", "poor", "below average", "average", "above average", "good”, or "excellent" chest muscular strength and endurance.

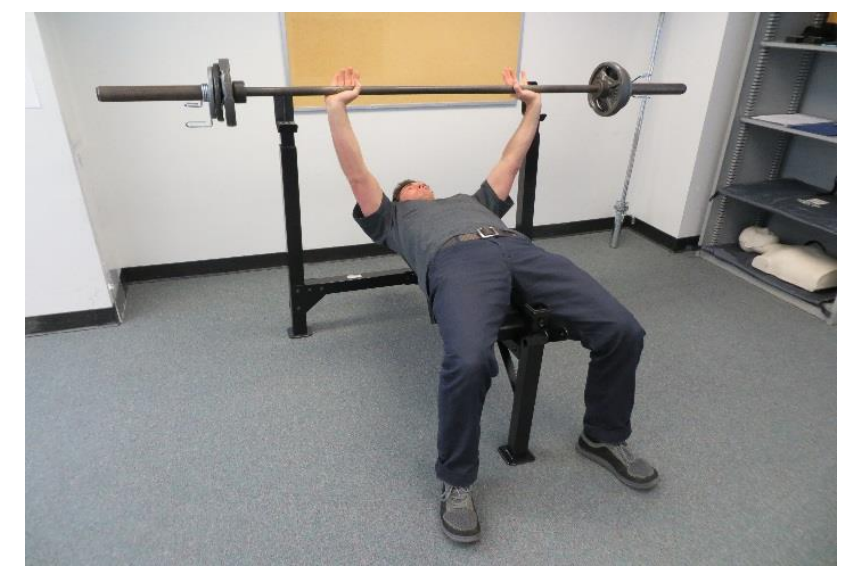

Figure 3. YMCA Bench Press Test.

After completion of the YMCA Bench Press Test, subjects were prepped using an alcohol wipe and cotton gauze, and 3 electrocardiography (ECG) electrodes were placed on the surface of the skin to permit collection of the electrocardiogram during the YMCA 
Step Test and Chest Compression Testing. The ECG was collected using Holter monitoring during tests in the Webb Human Performance Laboratory at Cal Poly.

\section{$\underline{\text { YMCA Step Test }}$}

The YMCA 3-minute step test was performed to evaluate the subject's pulse rate recovery for prediction of cardiorespiratory efficiency (Appendix E). Each subject was oriented to the testing protocol prior to the test. The YMCA step test requires the subject to step up and down using a 12-inch bench for three minutes at a prescribed stepping cadence of 24 steps per minute (Figure 4). Once the 3 minutes was complete, the subject

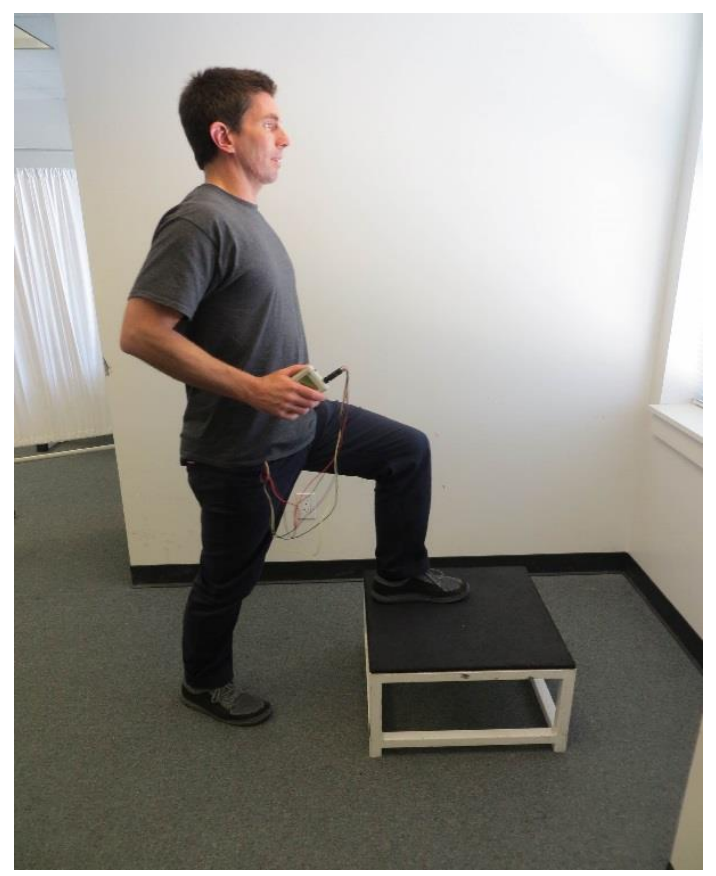

Figure 4. YMCA 3-Minute Step Test

was asked to be seated and remain still as an experimenter measured their heart rate for a 60 second period. The subject's heart rate score was then compared to scores from a reference chart within the subject's age bracket. This comparison determined whether the subject was categorized as having either "poor", "below average", "average", "good", or "excellent" cardio-respiratory fitness. 


\section{Chest Compression Tests}

Upon completion of the pre-test physical fitness evaluations experimenters explained the chest compression procedure on the manikin and allowed the subject to practice the proper form and get familiar with the lighting cues. American Heart Association (AHA) guidelines for performing CPR (Appendix F) were maintained by experimenter oversight. A review of proper compression form and technique was administered prior to performance of study trials. The next step was to randomly assign subjects into groups that performed compressions at varying rates. There was no critique of performance, or encouraging words, by experimenters, once subjects began a chest compression trial.

\begin{tabular}{|c|c|c|c|}
\hline Numeric Scale & Level of Exertion & Numeric Scale & Level of Exertion \\
\hline 0 & None & 5 & Intense \\
\hline .5 & Very, very light & 6 & \\
\hline 1 & Very light & 7 & Very intense \\
\hline 2 & Light & 8 & \\
\hline 3 & Moderate & 9 & Very, very intense \\
\hline 4 & A little intense & 10 & Maximum \\
\hline
\end{tabular}

Figure 5. RPE Scale. (Borg, 1982)

Holter monitoring was used to record the ECG rhythm and heart rate, and the subject's Rate of Perceived Exertion (RPE) was recorded three times, at each 5-minute 
interval, after the beginning of trial. The Borg (1982) RPE scale of 0-10, 0 being "nothing at all" and 10 being "very, very strong," was explained to the subjects prior to the beginning of the study. This new category scale with ratio properties allows for verbal expression to correlate with a number scale that is easy to interpret. The categorical expressions for each numeric level describe the intensity of physical exertion experienced by the subject. As the number increased toward 10, so did the overall physical exertion.

\section{$\underline{\text { Preston Manikin }}$}

Compressions were performed using a Preston CPR manikin with an adequate compression depth indicator (clicker). By use of indicator lights on the left shoulder of the Preston manikin (Figure 6), experimenters evaluated compression rate. One red light

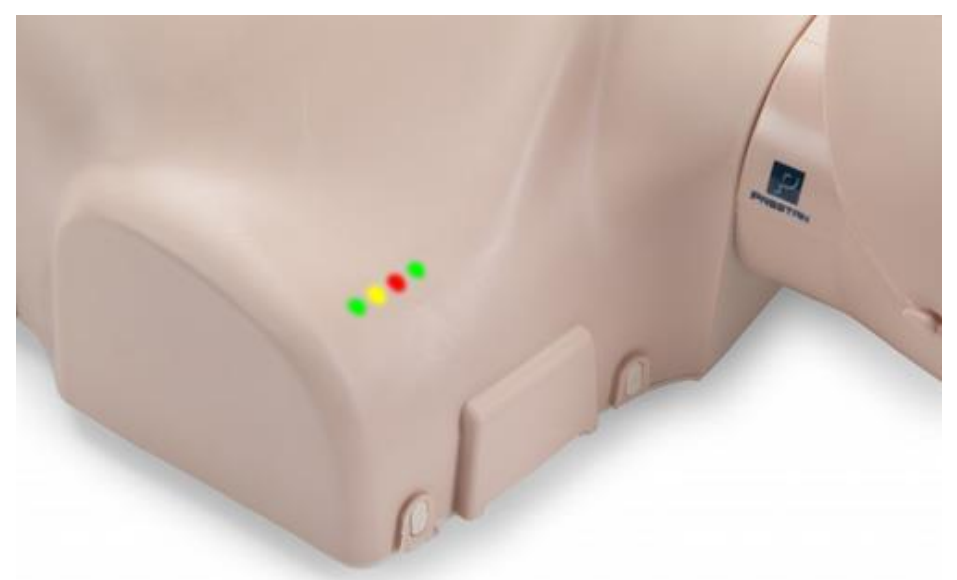

Figure 6. Light Biofeedback on Shoulder of CPR Manikin

indicated a compression per minute (cpm) rate of 1 to 59, one yellow light indicated 60 to $79 \mathrm{cpm}$, one green light indicated 80-99 cpm, and two green lights indicated a $\geq 100 \mathrm{cpm}$ rate. The following compression test termination criteria were used: 1 . Subject opted to discontinue compressions; 2. Subject failed to maintain a minimal trial compression rate $\geq 100 \mathrm{cpm}$ ( 2 green lights $)$ or $\geq 80 \mathrm{cpm}$ ( 1 green light) for more than 5 seconds; 
3. Subject compressed without producing a "click" 3 times in a row; or 4. Subject completed 15 minutes of compressions.

Layperson subjects (i.e., those without an occupational responsibility to perform CPR) were asked to meet separately at the Webb Human Performance lab in the Kinesiology building on Cal Poly State University campus at a time that was determined by both the subject and the investigators. Due to schedule conflicts concerning professional rescuers, testing procedures were administered at their place of business if this was more convenient for the subject.

The CPR manikin was placed on its back on a firm surface. Each subject was asked to kneel next to the manikin's neck and shoulders on a mat and place the heel of one hand over the center of the manikin's chest, between the nipples, and place the other hand on top of the first hand. Experimenters asked subjects to keep their elbows straight and position their shoulders directly above their hands (Figure 7, Appendix F). Subjects were asked to use their upper body weight (not just the arms) as they pushed straight down (compressed) on the chest at least 2 inches $(51 \mathrm{~mm})$. Subjects were asked to push hard. A Matrix MR 500 Quartz Metronome was used to maintain cadence at each compression rate.

Each subject was asked to perform 2 trials of compressions with at least 5-minute rest period between trials. Subjects performed one trial consisting of continuous compressions at a rate of $\geq 80 \mathrm{cpm}$, and another trial consisting of continuous compressions at a rate of $\geq 100 \mathrm{cpm}$. 


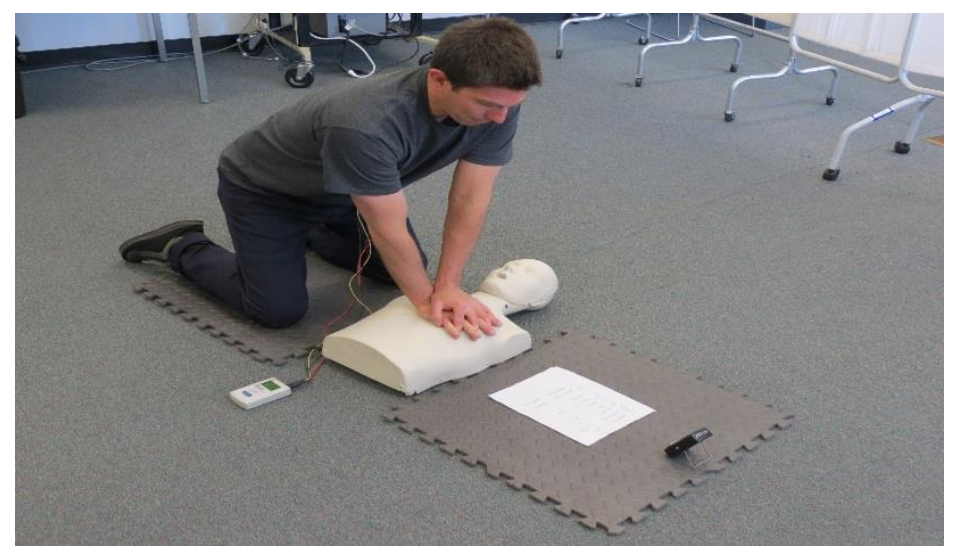

Figure 7. Chest Compressions on Manikin.

The compression rate trial order was randomized in order to control for a possible order effect (Figure 8). It was anticipated that fatigue and learning might influence the results during the second compression trial.

The baseline, peak, and terminal heart rates (HR) were determined from the ECG record for each trial and for each subject. The baseline HR was calculated using the slowest three beats within a few minutes of the start of each trial. The peak HR was the highest rate observed during each trial, and the terminal HR was the rate at the end of each trial. The differences in baseline, peak, and terminal HR between layperson and professional rescuers were evaluated.

After the collection of data the subjects were asked if they had any questions and were thanked for their participation in the study. Data were kept confidential; paper copies of records were kept in locked offices and computerized information was stored on password-protected computers. No names were used in the reports of this research without the subject's permission. Identifiers were destroyed after the results were analyzed, except on informed consent forms, which were maintained for a period of at least 2 years following data collection. 
Subjects

\begin{tabular}{l}
\hline 47 Lay Rescuers \\
13 Male \& 34 \\
Female
\end{tabular}

14 Professional

Rescuers

7 Male \& 7 Female $\underline{\text { Pre-Tests }}$

\begin{tabular}{|l|}
\hline Informed Consent \\
PAR-Q \\
History \& \\
Demographics \\
YMCA Bench Press \\
YMCA Step Test \\
\hline
\end{tabular}

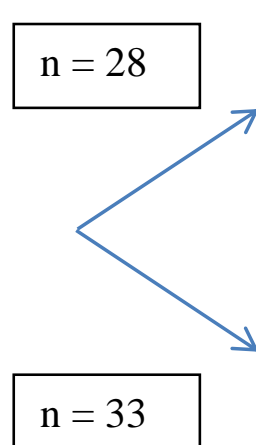

$\geq 80$ Compressions/min

$\longrightarrow \geq 100$ Compressions/min

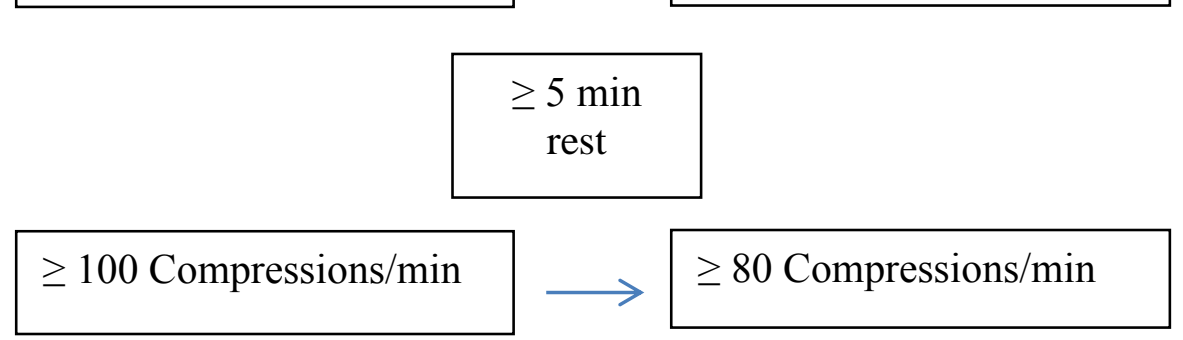

Figure 8. Test Procedures: Controlling for Order Effect 


\section{$\underline{\text { Statistical Analysis }}$}

The statistical procedures were selected based on consultation with Dr. Andrew Schaffner and Julia Goll. A Randomized Complete Block Design (Winer, Brown \& Michels, 1991), which groups experimental units into blocks in order to observe the true differences between, was used in this study, and collected data analyzed were by use of a logistic regression statistical method. Due to the dependent variable being categorical and having more than one independent variable, we found that logistic regression was best fit to analyze the data by using probability scores as the predicted values of the dependent variable. In order to balance the design each subject received the same number of treatments and each subject participated in all treatments. To incorporate a crossover design to the study, the sequence of each of the two treatments was changed for each subject. In this experimental study, due to the layperson and professional rescuers being the categorical explanatory variables and all the response variables (compression rate, time to fatigue, heart rate, and RPE) being quantitative, researchers wanted to analyze the difference between two or more populations. 


\section{Chapter 4}

\section{RESULTS AND DISCUSSION}

\section{$\underline{\text { Results }}$}

\section{Subjects}

Data were collected for this study between February 3, 2014, and March 14, 2014. The sample size of the study included 61 subjects, 20 men and 41 women (Table 2.). There were 13 men and 34 women for a total of 47 layperson rescuers. There were 14 professional rescuers including 7 men and 7 women. Most of the subjects $(43 / 61,70.5 \%)$, were currently certified to perform CPR. Of the 18 subjects that were not certified to perform CPR at the time of the study, the mean time since the last certification was 3.75 years. Overall, the average age for the population of the study was 24.5 years. The difference in the average age between men and women was 2 years (men: $25.8 \pm 8.6$ years; women: $23.8 \pm 4.2$ years). The mean age of male layperson subjects was $22.7 \pm 1.9$ years, range $=20$ to 27 years, and the mean age of female layperson subjects was $23.2 \pm 2.4$ years, range $=20$ to 31 years. The mean age of male professional subjects was $31.6 \pm 12.9$ years, range $=21$ to 56 years, and the mean age of female professional subjects was $27 \pm 8.6$ years, range $=21$ to 45 years. Demographic and other variables for these subjects are described in Table 2. The self-reported weights averaged $66.8 \mathrm{~kg}$, and the self-reported heights averaged $1.7 \mathrm{~m}$. The mean BMI based on self-reported weights and heights was 23.4.

\section{Fitness Tests}

Scores for YMCA Bench Press and Step Tests are presented in Table 3. Based on mean percentile scores for these tests this was a relatively fit cohort. The step test 
Table 2

Characteristics of Subjects

\begin{tabular}{|c|c|c|}
\hline Expertise & $\begin{array}{l}\text { Lay Rescuers } \\
(\mathrm{n}=47)\end{array}$ & $\begin{array}{l}\text { Professional Rescuers } \\
(n=14)\end{array}$ \\
\hline Mean Self-Reported Weight (kg) & $65.7 \mathrm{~kg}$ & $67.8 \mathrm{~kg}$ \\
\hline Mean Self-Reported Height (m) & $1.7 \mathrm{~m}$ & $1.7 \mathrm{~m}$ \\
\hline Mean BMI & 23.4 & 23.4 \\
\hline \multicolumn{3}{|l|}{ CPR Certification } \\
\hline $\begin{array}{l}\text { American Heart Association } \\
\text { American Red Cross }\end{array}$ & $\begin{array}{l}6 \\
23\end{array}$ & 7 \\
\hline Had Performed CPR on Real Victim & 0 & 6 \\
\hline \multicolumn{3}{|l|}{ Professional Rescuer Occupations } \\
\hline EMT & & 4 \\
\hline Paramedic & & 1 \\
\hline CNA, RN & & 2 \\
\hline $\mathrm{MD}, \mathrm{DO}$ & & 0 \\
\hline Lifeguard & & 6 \\
\hline Law Enforcement Officer & & 0 \\
\hline Exercise Physiologist & & 1 \\
\hline Other & & 0 \\
\hline
\end{tabular}


Table 3

YMCA Fitness Test Scores

\begin{tabular}{|c|c|c|}
\hline Expertise & $\begin{array}{l}\text { Lay Rescuers } \\
(\mathrm{n}=47)\end{array}$ & $\begin{array}{l}\text { Professional Rescuers } \\
(n=14)\end{array}$ \\
\hline \multicolumn{3}{|l|}{ Bench Press Test } \\
\hline \multicolumn{3}{|l|}{ Men (80 lb. bar) } \\
\hline Mean Repetitions & 28.2 & 31.1 \\
\hline Mean Percentile Score & $65.1 \%$ & $74.9 \%$ \\
\hline \multicolumn{3}{|l|}{ Women (35 lb. bar) } \\
\hline Mean Repetitions & 31.5 & 26.6 \\
\hline Mean Percentile Score & $72.9 \%$ & $66.5 \%$ \\
\hline \multicolumn{3}{|l|}{ Step Test } \\
\hline \multicolumn{3}{|l|}{ Men } \\
\hline Mean Post HR & 77.2 & 78.1 \\
\hline Mean Percentile Score & $86.6 \%$ & $89.9 \%$ \\
\hline \multicolumn{3}{|l|}{ Women } \\
\hline Mean Post HR & 82.5 & 83.6 \\
\hline Mean Percentile Score & $88.7 \%$ & $87.5 \%$ \\
\hline
\end{tabular}


percentile was calculated to determine an aerobic fitness difference between rescuers. The mean layperson rescuer aerobic percentile score was $88 \%$ and the professional rescuer was $95.5 \%$. The mean bench press percentile for layperson rescuers was $70.8 \%$ and the professional rescuer was $70.7 \%$.

\section{$\underline{\text { Chest Compression Tests }}$}

Out of the sample size of 61 subjects, 28 subjects performed trials with a compression rate of $\geq 80 \mathrm{cpm}$ before the trial of $\geq 100 \mathrm{cpm}$. There were 33 out of 61 subjects who performed the $\geq 100 \mathrm{cpm}$ rate before the trial of $\geq 80 \mathrm{cpm}$ rate. The duration of compression performance was evaluated in minutes and seconds and then converted to a decimal number up to two decimal places. Each trial of compressions was limited to a maximum duration of 15.0 minutes. Each subject completed one trial of $\geq 80$ compressions per minute $(\mathrm{cpm})$ and one trial of $\geq 100 \mathrm{cpm}$ until fatigue set in or 15.0 minutes was reached. The average duration of compressions, at a compression rate of $\geq$ $80 \mathrm{cpm}$, for layperson rescuers was 10.9 minutes. The average duration of compressions, at a compression rate of $\geq 80 \mathrm{cpm}$, for professional rescuers was 12.9 minutes. There was not enough evidence that these times were significantly different $(\mathrm{p}=.1696)$. For layperson rescuers, at a rate of $\geq 100 \mathrm{cpm}$, the average duration was 10.6 minutes. For professional rescuers at a rate of $\geq 100 \mathrm{cpm}$, the average duration was 12.8 minutes. There was not enough evidence that these times were significantly different $(\mathrm{p}=.1388)$. There were 11 out of the 14 (79\%) professional rescuers who were able to complete the 15 minute trial and seven of those eleven (64\%) were male rescuers. There were 24 out of the $47(51 \%)$ layperson rescuers who were able to complete the 15 minute trial and eight of those twenty-four (33\%) were male rescuers. 
The Rating of Perceived Exertion (RPE), on a scale from 0-10 (0 meaning no exertion at all; 10 meaning maximal exertion), was evaluated every 5 minutes until completion of trial (i.e., at minutes 5.0, 10.0 and 15.0). During the $\geq 80 \mathrm{cpm}$ rate, the average RPEs for layperson rescuers were 3.5, 3.8, and 3.4 respectively for each 5-minute period. For professional rescuers, at $\geq 80 \mathrm{cpm}$, the average RPEs were 3.3, 3.0, and 3.9, respectively. At the $\geq 100 \mathrm{cpm}$ rate, the average RPEs for layperson rescuers were 3.5, 4.2, and 3.9, respectively. For professional rescuers, at $\geq 100 \mathrm{cpm}$, the average RPEs were 3.4, 4.7, and 5.0, respectively. Overall, the mean RPE for both layperson and professional rescuers, at a rate of $\geq 80 \mathrm{cpm}$, was 5.2 . At the rate of $\geq 100 \mathrm{cpm}$, the mean RPE was 5.9. The overall RPEs for each 5-minute period, at a rate of $\geq 80 \mathrm{cpm}$, were 4.1, 5.5, and 6.1, respectively. At the $\geq 100 \mathrm{cpm}$ rate they were $4.4,6.5$, and 7.3, respectively.

\section{$\underline{\text { Time to fatigue }}$}

A logistic model was fit for subjects that performed 15 minutes of continuous chest compressions at a rate of $\geq 80$ per minute. The explanatory variables used in the model were male/female, professional/lay rescuer, treatment order, baseline heart rate at $\geq 80$ and $\geq 100 \mathrm{cpm}$, bench press percentile, step test percentile, and age. The output showed strong statistical evidence $(\mathrm{p}$-value $=.0115)$ that the predictors of this model affect the odds of completing the entire 15 minutes. Being a layperson rescuer decreased the odds of completing the whole 15 minutes $(\mathrm{p}=.0366$ ), after adjusting for all other variables in the model. Having a high bench press percentile score increased the odds of completing the entire 15 minutes $(\mathrm{p}=.0135)$, after adjusting for all other variables in the model. Being female decreased the odds of completing the entire 15 minutes, after 
adjusting for all other variables in the model $(\mathrm{p}=0.0451)$. There was $90 \%$ confidence that the odds of completing 15 minutes increased by a magnitude of at least 1.36 and at most 4.11 for males, after adjusting for all other variables in the model.

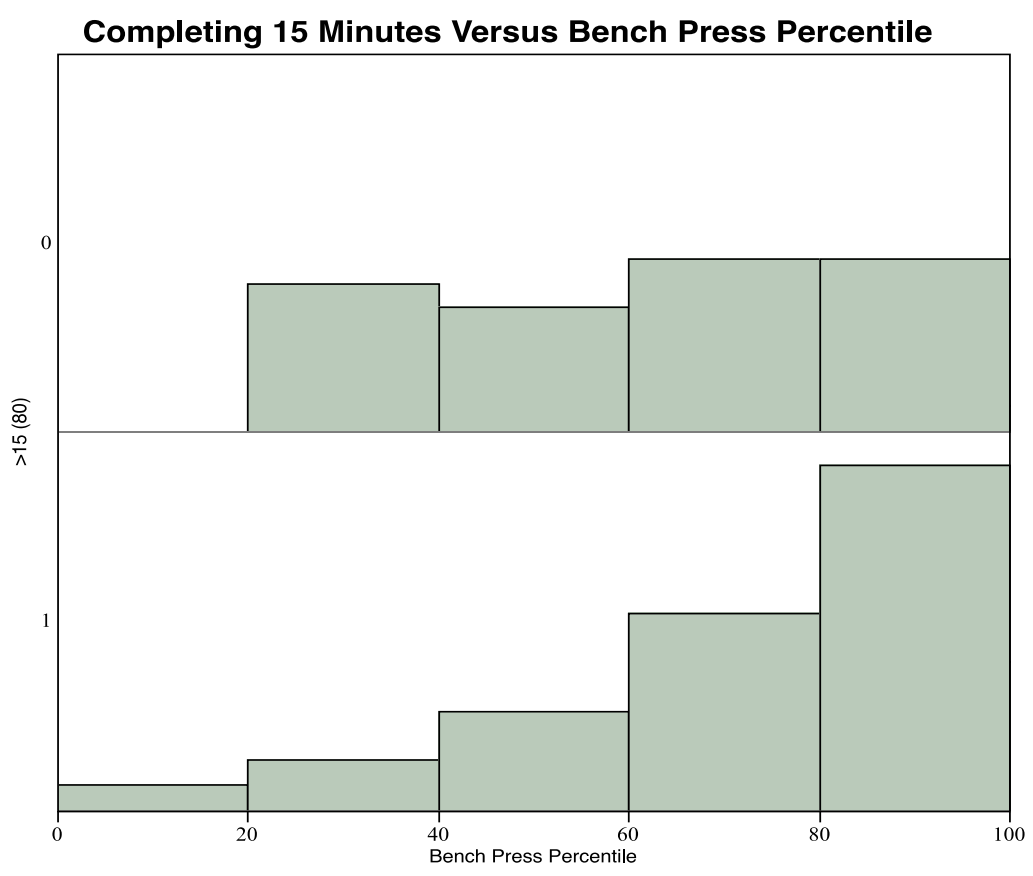

Figure 9. Bench Press Percentile Versus Reaching 15 Minutes at $\geq 80 \mathrm{cpm}$. On the $Y$ axis, the graph is split using (0) for not making it to 15 minutes and (1) for making it to 15 minutes.

A logistic fit statistical analysis was completed for subjects that performed 15 minutes of continuous chest compressions at a rate of $\geq 100$ per minute. It was concluded that the predictors of this model affected the odds of making it to the 15minute mark after adjusting for all other variables in the model $(\mathrm{p}=.0040)$. Being a layperson rescuer decreased the odds of completing the 15 minutes, after adjusting for all other variables in the model $(\mathrm{p}=.0346)$. Having a high bench press percentile score increased the odds of making it to 15 minutes $(\mathrm{p}=.0188)$. Being female decreased the 
odds of making it to the 15 minute time $(p=.0068)$. At $90 \%$ confidence, the odds of completing 15 minutes was increased by a magnitude of at least 3.02 and at most 66.37 for males, after adjusting for all other variables in the model.

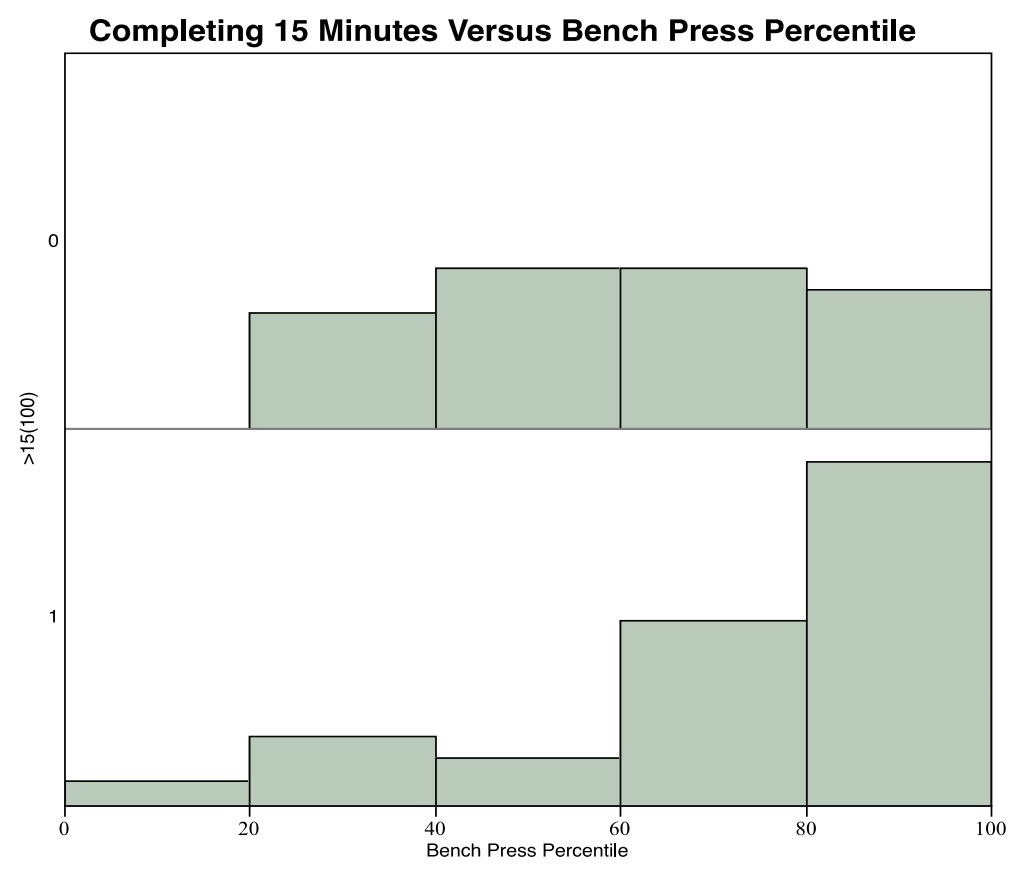

Figure 10. Bench Press Percentile Versus Reaching 15 Minutes at $\geq 100 \mathrm{cpm}$. On the Y axis, the graph is split using (0) for not making it to 15 minutes and (1) for making it to 15 minutes.

\section{$\underline{\text { Ratings of perceived exertion }}$}

In evaluating if the RPE means for each of the responses was different from one another, a multivariate analysis was used, fitting a MANOVA model, with predetermination to use Wilks' Lambda statistic. The perceived exertions between $\geq 80$ and $\geq 100 \mathrm{cpm}$ were not different at different time intervals $(5,10$, and 15 minutes), after adjusting for all other variables in the model $(\mathrm{p}=.0912)$. The mean perceived exertion between $\geq 80$ and $\geq 100 \mathrm{cpm}$ was not different for males and females, at time 5,10 , or 15 
minutes $(\mathrm{p}=.0668)$. Subjects did not experience different perceived exertion between $\geq$ 80 and $\geq 100 \mathrm{cpm}$, after adjusting for all other variables in the model $(\mathrm{p}=.2536)$.

Table 4. RPE Tabulated for 5, 10, and 15 Minutes at $\geq 80$ and $\geq 100 \mathrm{cpm}$.

\begin{tabular}{|c|c|c|c|c|c|}
\hline \multirow[t]{2}{*}{ Condition } & \multirow[t]{2}{*}{$\underline{\text { Statistic }}$} & \multicolumn{2}{|c|}{ Gender } & \multicolumn{2}{|c|}{ Proficiency } \\
\hline & & Female & Male & Lay & $\underline{\text { Pro }}$ \\
\hline \multirow{3}{*}{ RPE (5)(80) } & Mean & 4.41 & 3.64 & 4.32 & 3.55 \\
\hline & Std. Error & 0.29 & 0.47 & 0.27 & 0.59 \\
\hline & $\mathrm{n}$ & 32 & 19 & 38 & 13 \\
\hline \multirow[t]{3}{*}{ RPE (5)(100) } & Mean & 4.78 & 3.81 & 4.58 & 3.92 \\
\hline & Std. Error & 0.32 & 0.29 & 0.28 & 0.41 \\
\hline & $\mathrm{n}$ & 30 & 18 & 36 & 12 \\
\hline \multirow[t]{3}{*}{ RPE (10)(80) } & Mean & 6.15 & 4.56 & 6.09 & 3.86 \\
\hline & Std. Error & 0.41 & 0.53 & 0.39 & 0.45 \\
\hline & $\mathrm{n}$ & 23 & 17 & 29 & 11 \\
\hline \multirow[t]{3}{*}{ RPE (10)(100) } & Mean & 7.00 & 5.91 & 6.98 & 5.50 \\
\hline & Std. Error & 0.48 & 0.56 & 0.41 & 0.70 \\
\hline & $\mathrm{n}$ & 23 & 17 & 28 & 12 \\
\hline \multirow[t]{3}{*}{ RPE (15)(80) } & Mean & 6.70 & 5.27 & 6.60 & 4.95 \\
\hline & Std. Error & 0.50 & 0.57 & 0.47 & 0.60 \\
\hline & $\mathrm{n}$ & 20 & 15 & 24 & 11 \\
\hline \multirow[t]{3}{*}{ RPE (15)(100) } & Mean & 7.72 & 6.85 & 7.73 & 6.36 \\
\hline & Std. Error & 0.53 & 0.58 & 0.48 & 0.61 \\
\hline & $\mathrm{n}$ & 18 & 17 & 24 & 11 \\
\hline
\end{tabular}

\section{$\underline{\text { Heart rate }}$}

There were no differences between the heart rates for $\geq 100-\geq 80 \mathrm{cpm}$ at 5,10 , or 15 minutes, $(\mathrm{p}=.2350)$. 
Mean RPE Between Male and Female Rescuers

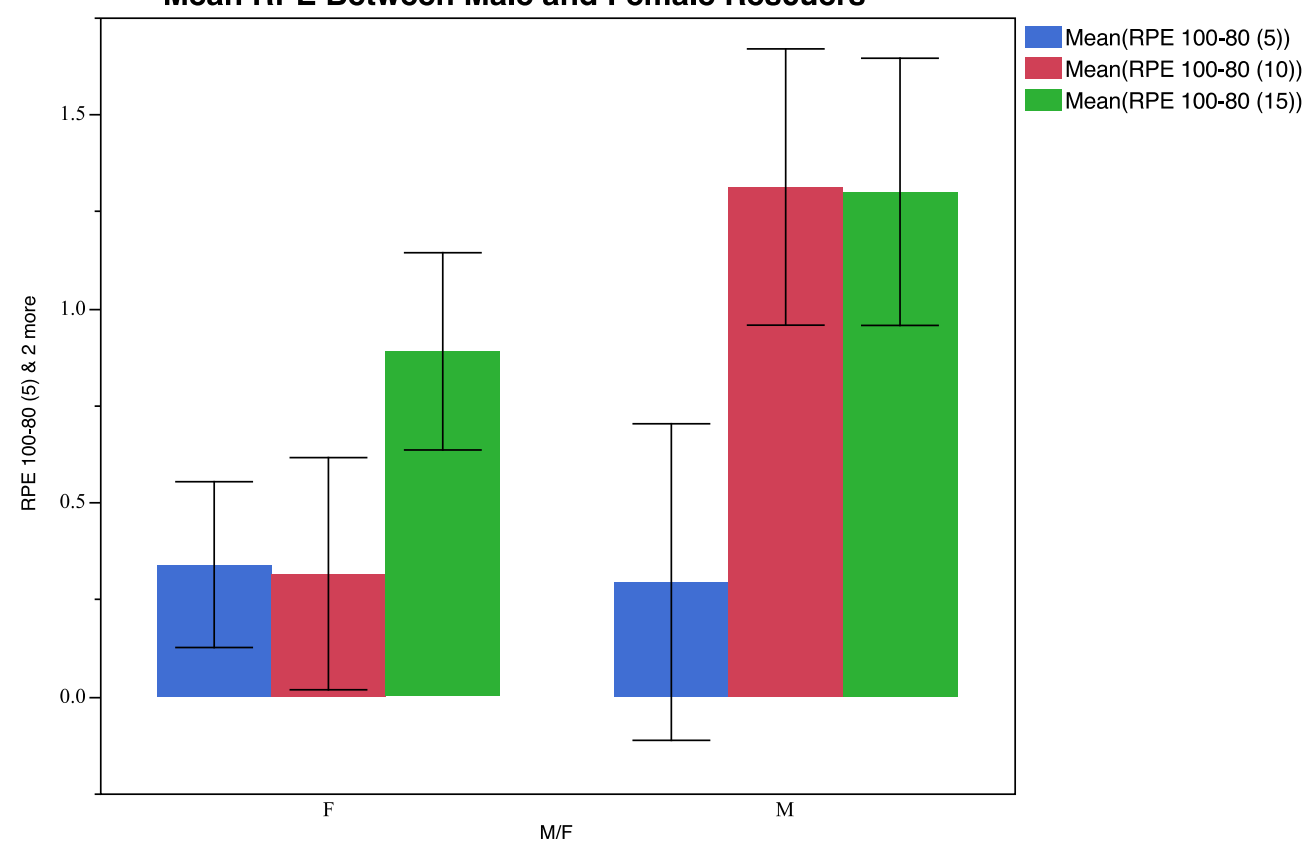

Figure 11. Average RPE at 5, 10, and 15 Minutes Versus M/F Subjects.

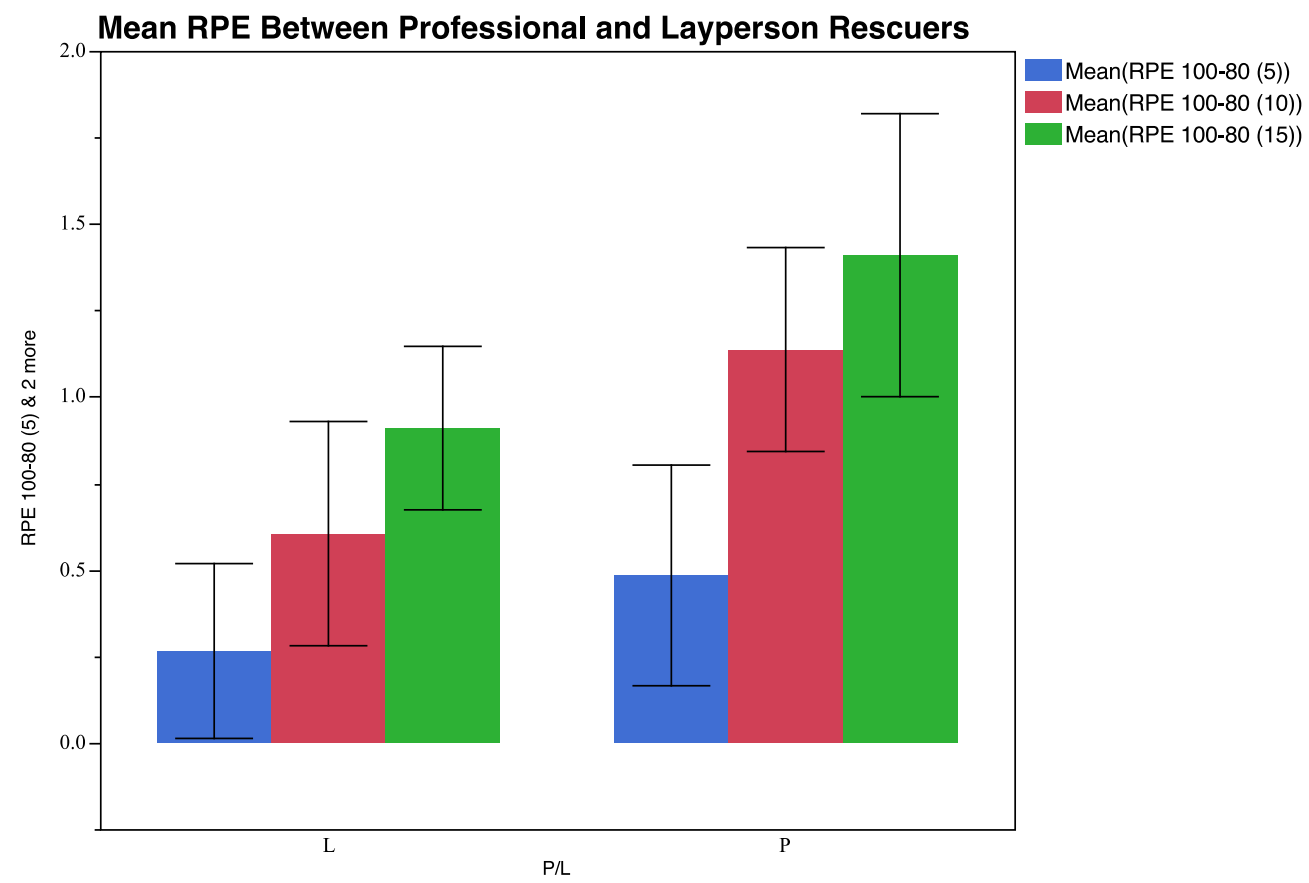

Figure 12. Average RPE at 5, 10, and 15 Minutes Versus P/L Subjects. 
Table 5. HR Tabulated for 5, 10, and 15 Minutes at $\geq 80$ and $\geq 100 \mathrm{cpm}$.

\begin{tabular}{|c|c|c|c|c|c|}
\hline \multirow[t]{2}{*}{ Condition } & \multirow[t]{2}{*}{$\underline{\text { Statistic }}$} & \multicolumn{2}{|c|}{ Gender } & \multicolumn{2}{|c|}{$\underline{\text { Proficiency }}$} \\
\hline & & Female & Male & Lay & $\underline{\text { Pro }}$ \\
\hline HR (5)(80) & $\begin{array}{l}\text { Mean } \\
\text { Std. Error } \\
\mathrm{n}\end{array}$ & $\begin{array}{l}128.03 \\
4.23 \\
30\end{array}$ & $\begin{array}{l}115.2 \\
3.87 \\
20\end{array}$ & $\begin{array}{l}125.82 \\
3.47 \\
38\end{array}$ & $\begin{array}{l}113.67 \\
6.11 \\
12\end{array}$ \\
\hline HR (5)(100) & $\begin{array}{l}\text { Mean } \\
\text { Std. Error } \\
\mathrm{n}\end{array}$ & $\begin{array}{l}130.14 \\
4.55 \\
29\end{array}$ & $\begin{array}{l}117.0 \\
4.93 \\
18\end{array}$ & $\begin{array}{l}127.83 \\
4.11 \\
35\end{array}$ & $\begin{array}{l}117.17 \\
6.16 \\
12\end{array}$ \\
\hline HR (10)(80) & $\begin{array}{l}\text { Mean } \\
\text { Std. Error } \\
\mathrm{n}\end{array}$ & $\begin{array}{l}133.43 \\
4.91 \\
21\end{array}$ & $\begin{array}{l}117.22 \\
4.65 \\
18\end{array}$ & $\begin{array}{l}127.64 \\
4.47 \\
28\end{array}$ & $\begin{array}{l}121.64 \\
6.16 \\
11\end{array}$ \\
\hline HR (10)(100) & $\begin{array}{l}\text { Mean } \\
\text { Std. Error } \\
\mathrm{n}\end{array}$ & $\begin{array}{l}133.48 \\
4.74 \\
23\end{array}$ & $\begin{array}{l}120.82 \\
5.47 \\
17\end{array}$ & $\begin{array}{l}129.89 \\
4.41 \\
28\end{array}$ & $\begin{array}{l}123.92 \\
6.80 \\
12\end{array}$ \\
\hline HR (15)(80) & $\begin{array}{l}\text { Mean } \\
\text { Std. Error } \\
n\end{array}$ & $\begin{array}{l}134.40 \\
5.01 \\
20\end{array}$ & $\begin{array}{l}118.07 \\
5.57 \\
15\end{array}$ & $\begin{array}{l}130.42 \\
4.98 \\
24\end{array}$ & $\begin{array}{l}120.82 \\
6.01 \\
11\end{array}$ \\
\hline HR (15)(100) & $\begin{array}{l}\text { Mean } \\
\text { Std. Error } \\
\mathrm{n}\end{array}$ & $\begin{array}{l}136.53 \\
5.54 \\
17\end{array}$ & $\begin{array}{l}121.65 \\
5.56 \\
17\end{array}$ & $\begin{array}{l}131.22 \\
5.18 \\
23\end{array}$ & $\begin{array}{l}124.64 \\
6.57 \\
11\end{array}$ \\
\hline
\end{tabular}




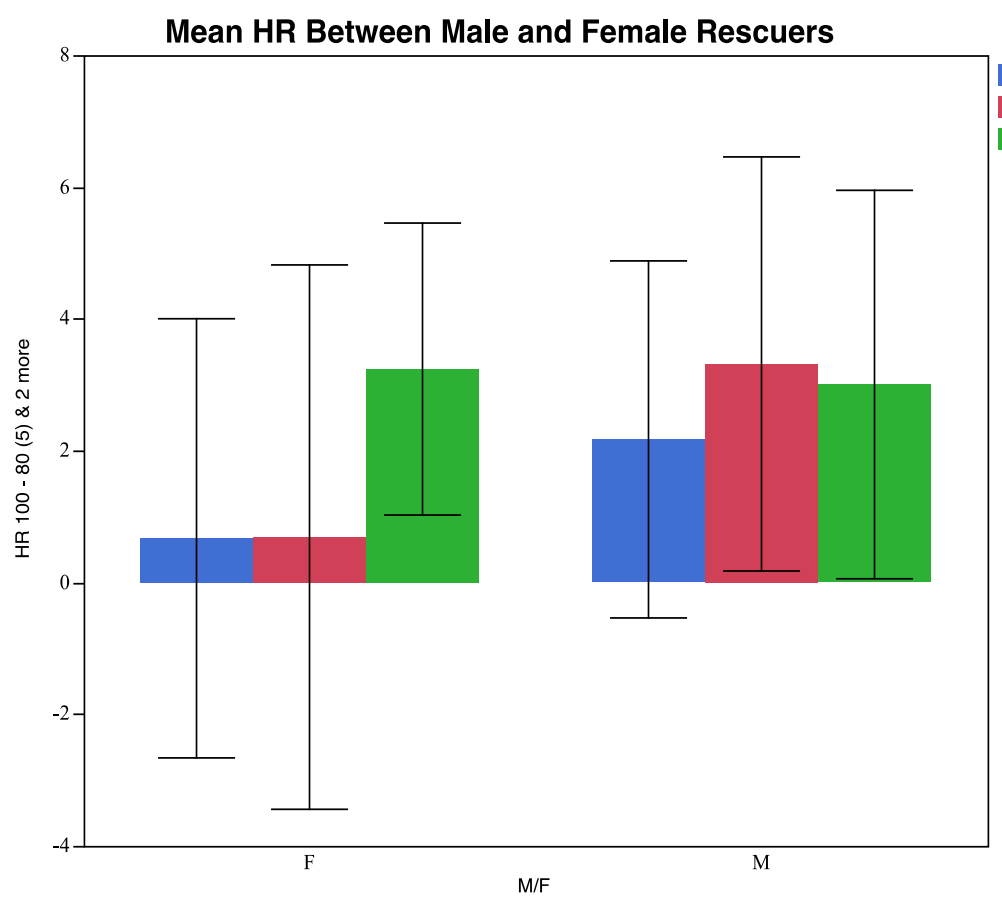

Mean(HR $100-80$ (5))

Mean(HR 100 - 80 (10))

Mean(HR $100-80(15))$

Figure 13. Average HR at 5, 10, and 15 Minutes Versus M/F Subjects.

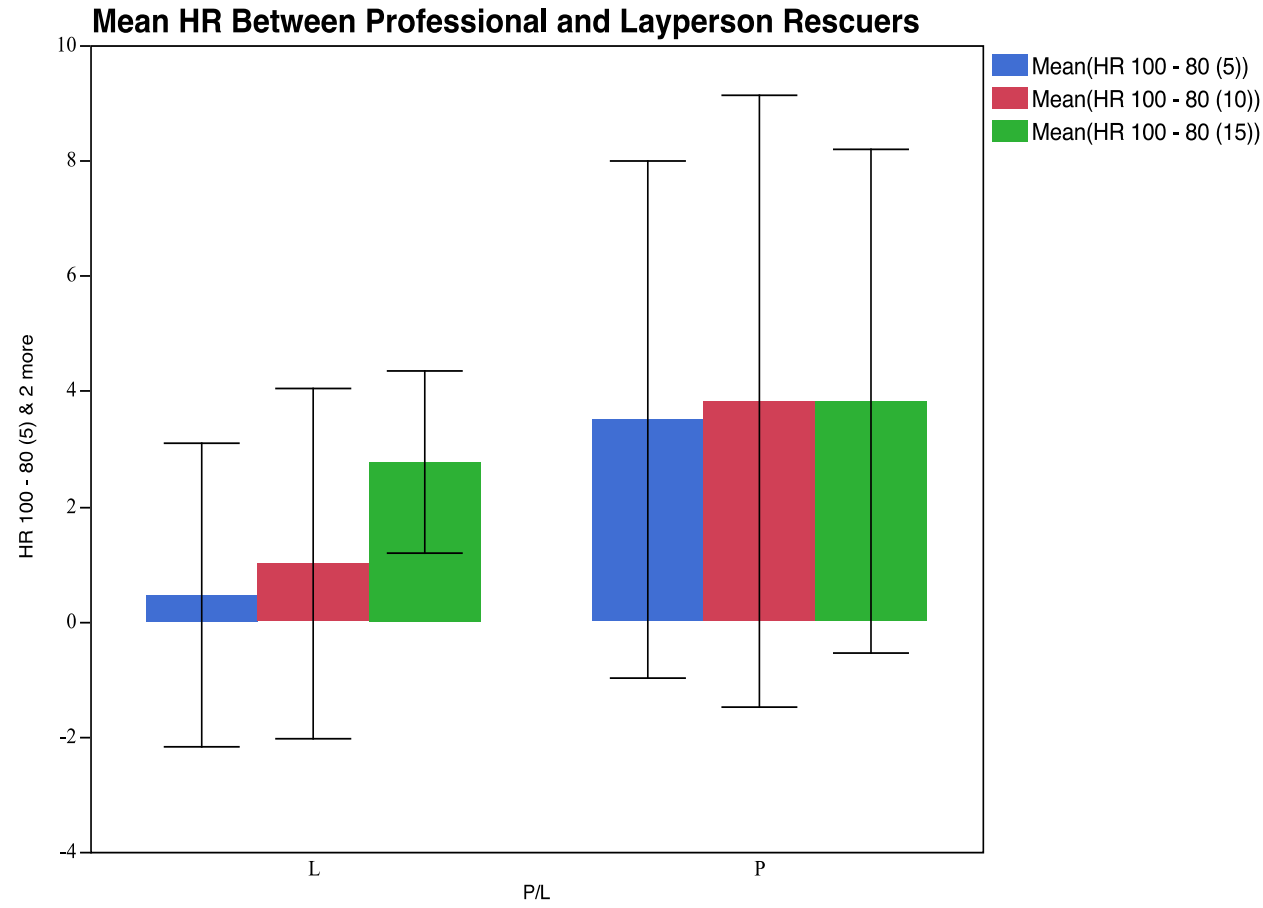

Figure 14. Average HR at 5, 10, and 15 Minutes Versus P/L Subjects. 


\section{$\underline{\text { Discussion }}$}

This study was developed to determine whether there was a correlation between the physical fitness of the rescuer and the rescuers ability to perform chest compressions for an extended period of time. A correlation between these two variables would allow researchers to better understand what is needed to alleviate the symptoms of fatigue and maximize a rescuer's ability to continue life saving procedures with the patient. The following paragraphs discuss some reasons which may have led to the results of this study.

\section{Interpretation of Results}

Professional rescuers successfully sustained compressions longer than the lay rescuer during the $\geq 100 \mathrm{cpm}$ rate, but not the $\geq 80 \mathrm{cpm}$ rate. Researchers have suggested that professional rescuers maintain a level of proficiency because they perform chest compressions as an occupational activity. The necessary endurance level of the professional rescuer in sustaining a high compression rate of $\geq 100 \mathrm{cpm}$ allowed them the ability to outlast the lay rescuers.

Male subjects were able to sustain compressions longer than female subjects during the $\geq 100 \mathrm{cpm}$ rate, but not the $\geq 80 \mathrm{cpm}$ rate. Male subjects, in comparison to female subjects, may have relied heavily on upper body strength to maintain a longer compression time at the higher rate of $\geq 100 \mathrm{cpm}$. Perhaps professional rescuers' chest strength has adapted to the demands of on-the-job CPR. Men naturally have the ability to acquire larger muscle mass in the upper body, compared to females, which might give them an advantage in performing compressions for a longer period of time. Those in a position to perform chest compressions on a regular basis would benefit from a routine of 
upper body muscular training. Females are encouraged, especially those in the Emergency Medical Services, to maintain a regular upper body strength training program with use of the bench press. Individuals who reside in rural areas, were EMS response times might be 15 minutes or longer, would benefit from a regular upper body strength program.

Subjects perceived that the exertion felt during chest compressions increased going from 5 to 10 to 15 minutes. Male subjects showed a higher perceived exertion at minute 10 and minute 15 compared to female subjects. Males having a higher exertion level might be due to activating upper body muscles early in the compression trial. By not utilizing other means, such as body weight, the muscles activated for each compression may lead to muscle fatigue and a higher perceived exertion. Professional rescuers perceived their exertion level to be higher than the lay rescuers at each time interval $(5,10$, and 15 minutes). Perhaps a lack of experience in performing compressions for extended periods of time, because professional rescuers rotate frequently, explains why professional rescuers perceived their exertion to be higher. Professional rescuers might have a better idea, compared to lay rescuers, of what it takes to perform compressions which in turn might hyperinflate their perception of what is to come as the length of the trial continues.

Even though there was a slight difference in the perception of exertion during compressions, there was no significant difference in heart rate for all responses tested between $\geq 100$ and $\geq 80 \mathrm{cpm}$. Disk corruption, during the testing process, led to researchers being unable to recover lost data. This disruption in the data processing might have might led to a lack of significant differences in heart rate between subjects. 
Selection of a majority of subjects of a certain age and fitness category from the university campus might have had an influence in heart rate data being similar between groups.

Bench press percentile showed a positive correlation to the duration of compressions. Researchers determined that a correlation between male subjects and the bench press percentile indicated that upper body strength is important for maintaining compressions for an extended period of time. The use of similar muscle groups, in comparing the bench press test to cardiocerebral resuscitation, may be a reason why a high bench press percentile would allow a rescuer to increase their compression duration.

In a study by Hansen et al. (2012) researchers showed a continued increase in lactate concentrations during 15 minutes of continuous compressions. As a result, this finding predicts that compressions can only be sustained for an extended period of time when lactic acid production can be tolerated, as with the addition of a greater maximal muscular strength (anaerobic capacity). These researchers' findings support the present study showing that upper body muscular strength has a relationship to compression duration. A study by Ock et al. (2011) had similar findings and suggested that muscle strength training be implemented to improve the quality of compression performance.

The same study by Ock et al. (2011) found that heart rate was not correlated to cardiocerebral resuscitation (CCR) quality which helps support the finding in the present study that heart rate did not vary significantly at different times during compression trials or between the different compressions rates.

There is correlation between a rescuer's upper body muscular strength and their ability to perform cardiocerebral resuscitation on a patient for at least 15 minutes. A 
regular strength training program, with emphasis on the bench press, might yield a compression duration of longer than 15 minutes. The results of this study highlight another valuable research outcome that can possibly help rescuers be better prepared for situations involving cardiocerebral resuscitation.

There were various reasons given by subjects for discontinuing compression trials (Figure 15). The most frequent complaint was that the subject experienced wrist

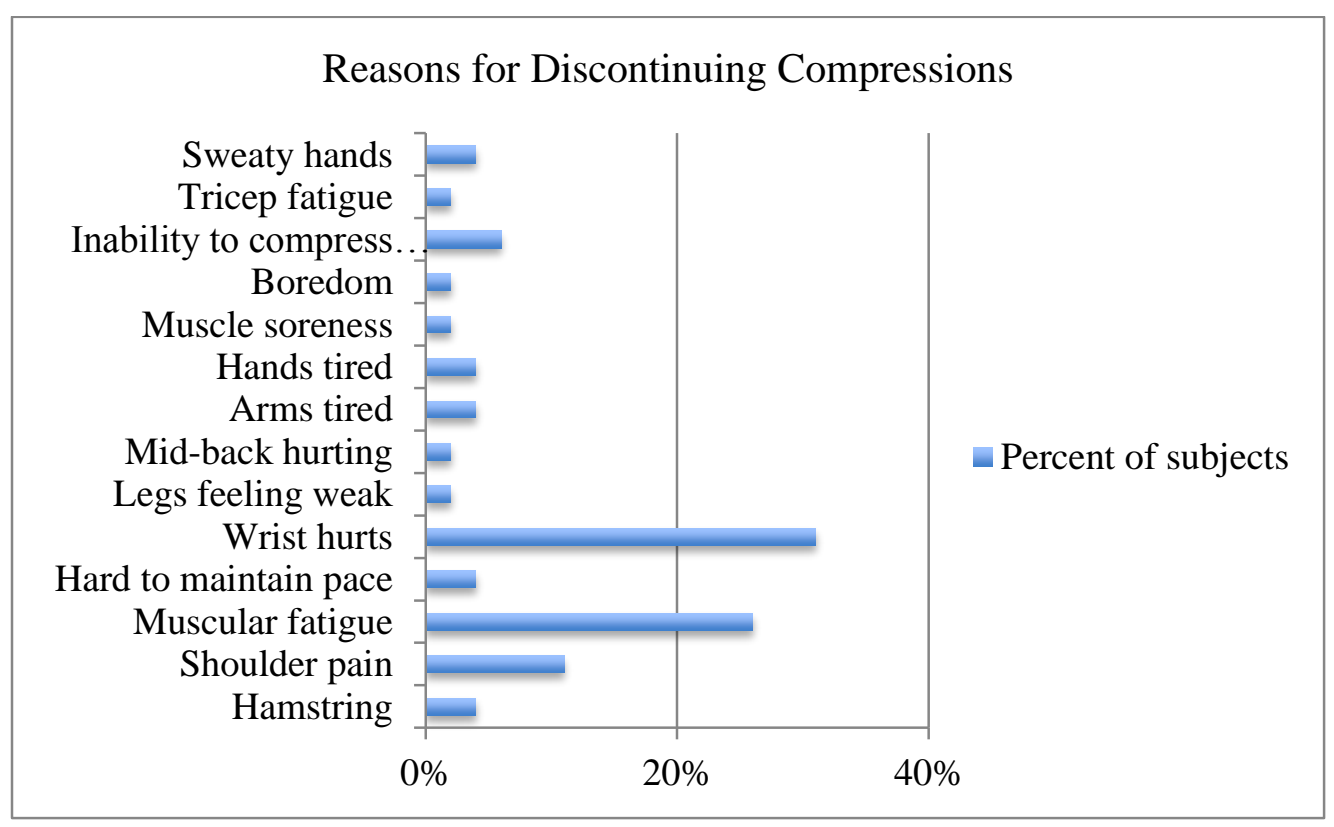

Figure 15. Reasons for Discontinuing Chest Compressions Before Completing 15 Minutes.

discomfort which made it difficult to continue compressions. Aside from the subjects experiencing sweaty hands, or boredom due to the repetitive nature of the trial, the majority of complaints centered around some form of muscular fatigue. The difficulty in maintaining pace of compressions and inability to compress deep enough were due to 
muscular weakness. Complaints were made regarding parts of the body that had key muscles used in the performance of chest compressions. Subjects complained about the legs (e.g., hamstrings), the wrists, hands, arms (e.g., triceps), and shoulders, as well as the mid-back. These muscular areas are all used to maintain proper form while performing chest compressions. 


\section{Chapter 5}

\section{SUMMARY, CONCLUSIONS, AND RECOMMENDATIONS}

\section{Summary}

The present study intended to describe fatigue rates at $\geq 80$ versus $\geq 100$ compressions per minute, and verify the utility of the current CPR guidelines for single rescuers among laypersons versus professionals, men versus women of varying ages, and persons of varying chest strength/endurance and cardiorespiratory fitness.

The present study was designed to evaluate the subject's pre-test physical fitness level and compare the test results with the performance of each compression trial. These data allowed researchers to determine a relationship between the subject's aerobic and anaerobic conditioning and their time to fatigue. Each subject was monitored, throughout the trial, for HR and RPE levels, which were used to chart the effect that continuous compressions had on each subject. These data allowed researchers to better understand how fatigue plays a part in the ability to sustain compressions for 15 minutes.

At first thought it seemed obvious that $\geq 100 \mathrm{cpm}$ would cause a greater rate of fatigue than the $\geq 80 \mathrm{cpm}$, yet this study showed a lack of statistically significant differences between the two compression rates. Researchers first hypothesized that the professional rescuers would have greater muscular and cardiorespiratory fitness, lower HR and RPE scores, and would have a lower level of fatigue compared to the lay rescuers. Upon evaluating the data it became evident that the differences were less obvious than previously thought.

This study was designed to evaluate how much of an effect physical fatigue had on the quality of chest compressions. This research highlights the need, in both 
professional and layperson CPR, for improved physical fitness in adapting to the increasing compression rates. The output showed a statistical correlation between upper body muscular strength, the performance of the bench press, and a rescuer's ability to improve compression duration.

The present study proposed that professional rescuers would perceive their exertion level to be lower than the layperson rescuers. In contrast, the professional rescuers perceived their exertion level to be higher than the layperson rescuers throughout the trials. Also contradictory to the proposed hypothesis was that male rescuers perceived their level of exertion to be higher than the female rescuers.

Proposed hypotheses for time to fatigue were supported as being a female, layperson rescuer decreased the odds of completing the entire 15 minute trial. Also, having a high bench press percentage increased the odds of completing compressions for the entire 15 minutes.

With the majority of sudden cardiac arrests occurring at home, an undetermined arrival time of emergency personnel, and ALS being affected by levels of perfusion, it is important to recognize the role that physical fitness plays in the performance of chest compressions and possibly the outcome of the patient. 


\section{$\underline{\text { Conclusions }}$}

1. Mean heart rate was not greater at $\geq 100 \mathrm{cpm}$ than $\geq 80 \mathrm{cpm}$.

2. Perceived exertions were not different between $\geq 100$ and $\geq 80 \mathrm{cpm}$ at the different time intervals of 5, 10 and 15 minutes.

3. There was no difference between the mean duration of compressions at $\geq 80 \mathrm{cpm}$ and $\geq 100 \mathrm{cpm}$.

4. Treatment order did not affect compression duration.

5. Treatment order did not affect HR.

6. Treatment order did not affect RPE.

7. Professional rescuers had greater endurance than lay rescuers at $\geq 100 \mathrm{cpm}$, but not at $\geq 80 \mathrm{cpm}$.

8. Professional rescuers did not have lower RPEs than lay rescuers.

9. Professional rescuers did not have different mean HRs than lay rescuers.

10. Men had greater endurance than women at $\geq 100 \mathrm{cpm}$, but not at $\geq 80 \mathrm{cpm}$.

11. Between $\geq 100$ and $\geq 80 \mathrm{cpm}$, males noticed a bigger difference in RPE than women at 5, 10, and 15 minutes.

12. There was no difference in mean HR between male and female rescuers at $\geq 100-\geq 80 \mathrm{cpm}$.

13. There is a relationship between YMCA bench press test score/upper body strength/endurance and compression duration.

14. There is no relationship between YMCA step test/cardiorespiratory fitness and compression duration. 


\section{$\underline{\text { Recommendations }}$}

\section{Future Research}

Recommendations for further research should focus on the degree to which the performance of the bench press affects the duration of chest compressions. A separate study evaluating subjects as they maintain a strength training program and an examination of compression duration to exhaustion would allow for researchers to get a better idea of how much upper body strength plays a part in fatigue.

A closer evaluation of male and female professional rescuers could shed light on not only the difference in fatigue between the sexes, but on how much upper body muscular endurance can have an influence in compression duration.

\section{Performance of Cardiocerebral Resuscitation}

In light of this conclusive data on the bench press having a significant influence on compression duration, it would be of value to suggest that the CPR certification process involve a physical fitness evaluation with an emphasis on bench press performance. Personnel in the emergency services, whose job description involves the possibility of performing chest compressions, would benefit from a regular strength training program that incorporates the use of the bench press as a means of improving compression duration.

Researchers recommend that rescuers use a metronome to pace compressions during cardiocerebral resuscitation. A metronome allows the rescuer to maintain a certain compression per minute rate and might help with the fatigue aspect over time.

Since wrist fatigue or discomfort of the rescuer was determined to be the most prevelant reason for discontinuing compressions, researchers suggest using a glove or 
brace to help prevent discomfort and to support the joint during extended compression trials.

The patient and the rescuer would benefit from staging advanced care providers in rural areas allowing for a less than 15 minute response time to potential patients in cardiac arrest. 


\section{REFERENCES}

Abella, B. S., Sandbo, N., Vassilatos, P., Alvarado, J. P., O’Hearn, N., Wigder, H. N., Hoffman, P., Tynus, K., Vanden Hoek, T. L., \& Becker, L. B. (2005). Chest compression rates during cardiopulmonary resuscitation are suboptimal: A prospective study during in-hospital cardiac arrest. Circulation. 111(4), 428-34.

American College of Sports Medicine. (2014). ACSM's guidelines for exercise testing and prescription $\left(9^{\text {th }}\right.$ ed.). Philadelphia: Wolters Kluwer/Lippincott Williams \& Wilkins, p. 34.

American Heart Association. (2011). Highlights of the 2010 american heart association guidelines for cpr and ecc, 8. Retrieved from: http://www.heart.org/idc/groups/heartpublic/@wcm/@ecc/documents/downloadable/ucm_317350.pdf

American Heart Association. (2013). Chain of survival. Retrieved from: http://www.heart.org/HEARTORG/CPRAndECC/WhatisCPR/ECCIntro/Chainof-Survival_UCM_307516_Article.jsp

Ashton, A., McCluskey, A., Gwinnutt, C. L., Keenan, A. M. (2002). Effect of rescuer fatigue on performance of continuous external chest compressions over 3 min. Resuscitation. 55(2), $151-155$. 
Berg, R. A., Hemphill, R., Abella, B. S., Aufderheide, T. P., Cave, D. M., Hazinski, M. F., Lerner, E. B., Rea, T. D., Sayre, M. R., Swor, R. A. (2010). American heart association guidelines for cardiopulmonary resuscitation and emergency cardiovascular care science. Part 5: Adult basic life support. Circulation, $122,5685-5705$.

Betz, A.E., Callaway, C.W., Hostler, D., Rittenberger, J.C. (2008). Work of cpr during two different compression-to-ventilation ratios with real-time feedback. Resuscitation. 79(2), 278-282.

Blackwell T. H., Kaufman J. S. (2002). Response time effectiveness: Comparison of response time and survival in an urban emergency medical services system. Academic Emergency Medicine, 9(4), 288-295.

Blackwell T. H., Line J. A., Willis J., Hicks G. M. (2009). Lack of association between prehospital response times and patient outcomes. Prehospital Emergency Care. $13,444-450$.

Blanchard I. E., Doig C. J., Hagel B. E., Anton A. R., Zygun D. A., Kortbeek J. B., Powell D. G., Williamson T. S., Fick G. H., \& Innes G. D. (2012). Emergency medical services response time and mortality in an urban setting. Prehospital Emergency Care, 16, 142-151.

Borg, G.A.V. (1982). Psychophysical bases of perceived exertion. Medicine and Science in Sport and Exercise, 14(5), pp.377-381. 
Campbell J. P., Gratton M. C., Salomone J. A., Lindholm D. J., \& Watson W. A.

(1994). System implications of the ambulance arrival-to-patient contact interval on response interval compliance. Prehospital and Disaster Medicine, 9(4), $230-233$.

Centers for Disease Control and Prevention. (2014). National Center for Chronic Disease Prevention and Health Promotion, Division for Heart Disease and Stroke Prevention. Heart disease facts. Retrieved from: http://www.cdc.gov/heartdisease/facts.htm

Cobb L. A., Fahrenbruch C. E., Walsh, T. R., Compass, M. K,, Olsufka, M., Breskin, B, \& Hallstrom, A. P. (1999). Influence of cardiopulmonary resuscitation prior to defibrillation in patients with out-of- hospital ventricular fibrillation. Journal of the American Medical Association, 281, 1182-1188.

Cunningham, L. M., Mattu, A., O’Connor, R. E., \& Brady, W. J. (2012). Cardiopulmonary resuscitation for cardiac arrest: The importance of uninterrupted chest compressions in cardiac arrest resuscitation. American Journal of Emergency Medicine, 30, 1630-1638.

De Maio, V. J., Stiell, I. G., Wells, G. A., \& Spaite, D. W. (2003). Optimal defibrillation response intervals for maximum out-of-hospital cardiac arrest survival rates. Annals of Emergency Medicine, 42(2), pp. 242-250.

Do, Y. K., Foo, K., Ng, Y. Y., \& Ong, M.E.H. (2013). A quantile regression analysis of ambulance response time. Prehospital Emergency Care, 17, 170-176. 
Feneley, M. P., Maier, G. W., Kern, K. B., Gaynor, J. W., Gall, S. A., Sanders, A. B., Raessler, K., Muhlbaier, L. H., Rankin, S., \& Ewy, G. A. (1988). Influence of compression rate on initial success of resuscitation and $24 \mathrm{hr}$. survival after prolonged manual cardiopulmonary resuscitation in dogs. Circulation, 77(1), 240-250.

Field, R. A., Soar, J., Davies, R. P., Akhtar, N., \& Perkins, G. D. (2011). The impact of chest compression rates on quality of chest compressions - a manikin study. Resuscitation, 83, 360-364.

Gold, L. S., Fahrenbruch, C. E., Rea, T. D., Eisenberg, M .S. (2010). The relationship between time to arrival of emergency medical services (EMS) and survival from out-of-hospital ventricular fibrillation cardiac arrest. Resuscitation, 81, $622-625$.

Hansen, D., Vranckx, P., Broekmans, T., Eijnde, B. O., Beckers, W., Vandekerckhove, P., Broos, P., \& Cendale, P. (2012). Physical fitness affects the quality of single operator cardiocerebral resuscitation in healthcare professionals. European Journal of Emergency Medicine, 19(1), 28 -34.

Heidenreich, J., Berg, R., Higdon, T., Ewy, G., \& Kern, K. (2006). Rescuer fatigue: Standard versus continuous chest-compression cardiopulmonary resuscitation. Academic Emergency Medicine, 13(10), 1020-1026.

Hightower, D., Thomas, S.H., Stone, C.K., Dunn, K., March, J.A. (1995). Decay in quality of closed-chest compressions over time. Annals of Emergency Medicine, 26(3), 300-303. 
Idris, A. H., Guffey, D., Aufderheide, T. P., Brown, S., Morrison, L. J., Nickols, P., Powell, J., Daya, M., Bigham, B. L., Atkins, D. L., Berg, R., Davis, D., Stiell, I., Sopko, G., Nichol, G., \& the Resuscitation Outcomes Consortium (ROC) Investigators. (2012). Relationship between chest compression rates and outcomes from cardiac arrest. Circulation, 125, 3004-3012.

Kern, K. B., Carter, A. B., Showen, R. L., Voorhees, W. D., Babbs, C. F., Tacker, W. A., \& Ewy, G. A. (1986). Twenty-four hour survival in a canine model of cardiac arrest comparing three methods of manual cardiopulmonary resuscitation. Journal of the American College of Cardiology, 7, 859-67.

Kern, K. B., Hilwig, R. W., Berg, R. A., Sanders, A. B., \& Ewy, G. A. (2002). Importance of continuous chest compressions during cardiopulmonary resuscitation: Improved outcome during a simulated single lay-rescuer scenario. Retrieved from: http://circ.ahajournals.org/

Kern, K. B., Sanders, A. B., Raife, J., Milander, M. M., Otto, C. W., \& Ewy, G. A. (1992). A study of chest compression rates during cardiopulmonary resuscitation in humans: The importance of rate-directed chest compressions. Archives of Internal Medicine, 152, 145-149.

Maier, G. W., Newton, J. R., Wolfe, J. A., Tyson, G. S., Olsen, C. O., Glower, D. D., Spratt, J. A., Davis, J. W., Feneley, M. P., \& Rankin, J. S. (1986). The influence of manual chest compression rate on hemodynamic support during cardiac arrest: high-impulse cardiopulmonary resuscitation. Circulation, 74(6)(2), 51-9. 
Manders, S., Geijsel, F. E. (2009). Alternating providers during continuous chest compressions for cardiac arrest: Every minute or every two minutes? Resuscitation, 80(9), 1015-8.

Mayo Foundation for Medical Education and Research. (2012). Sudden cardiac arrest: Risk factors. Retrieved from: http://www.mayoclinic.com/health/suddencardiac-arrest/DS00764/DSECTION=risk-factors

McDonald, C., Heggie, J., Jones, C., Thorne, C., \& Hulme, J. (2013). Rescuer fatigue under the 2010 erc guidelines, and its effect on cardiopulmonary resuscitation (cpr) performance. Emergency Medicine Journal, 30(8), pp. 623627.

Merriam-Webster (2013). Dictionary. An encyclopedia britannica company. Retrieved from: http://www.merriam-webster.com/

Monsieurs, K. G., Regge, M. D., Vansteelandt, K., Smet, J. D., Annaert, E., Lemoyne, S., Kalmar, A. F., \& Calle, P. A. (2012). Excessive chest compression rate is associated with insufficient compression depth in prehospital cardiac arrest. Resuscitation, 83(11), 1319-1323.

Mosby's Medical Dictionary. (2009). Elsevier. $8^{\text {th }}$ edition. Retrieved from: http://medical-dictionary.thefreedictionary.com/

National Institutes of Health. (2013). Turning discovery into health. Retrieved from: http://www.nhlbi.nih.gov

Narad, R. A., \& Driesbock K. R. (1999). Regulation of ambulance response times in California. Prehospital Emergency Care, 3(2), 131-135. 
Nichol, G., Detsky, A. S., Stiell, I. G., O’Rourke K., Wells G., \& Laupacis A. (1996). Effectiveness of emergency medical services for victims of out-of-hospital cardiac arrest: A metaanalysis. Annals of Emergency Medicine, 27, 700-710.

Ochoa, F. J., Ramalle-Gomara, E., Lisa, V., Saralegui, I. (1998). The effect of rescuer fatigue on the quality of chest compressions. Resuscitation, 37(3), 149-152.

Ock, S., Kim, Y., Chung, J. H., \& Kim, S. H. (2011). Influence of physical fitness on the performance of 5-minutes of continuous chest compression. European Journal of Emergency Medicine, 18(5), 251 -256.

Peleg K., \& Pliskin J. S. (2004). A geographic information system simulation model of ems: Reducing ambulance response time. American Journal of Emergency Medicine, 22(3), 164-170.

Pons, P. T., Haukoos, J. S., Bludworth, W., Cribley, T., Pons, K. A., Markouchick, V. J. (2005). Paramedic response time: Does it affect patient outcome? Academic Emergency Medicine, 12(7), 594-600.

Riera, S., Gonzalez, B.S., Alvarez, J.T., Fernandez, M.F., Saura, J.M. (2006). The physiological effect on rescuers of doing 2 min of uninterrupted chest compressions. Resuscitation, 74(1), 108 -112. 
Russo, S. G., Neumann, P., Reinhardt, S., Timmermann, A., Niklas, A., Quintel, M., \& Eich, C. B. (2011). Impact of physical fitness and biometric data on the quality of external chest compression: A randomized, crossover trial. BMC Emergency Medicine, 11, p. 20.

Salvucci, A., Kuehl, A., \& Clawson, J. J. (2004). The response time myth: Does time matter in responding to emergencies? Topics in Emergency Medicine, 26(2), 86-92.

Trowbridge, C., Parekh, J. N., Ricard, M. D., Potts, J., Patrickson, C., \& Cason, C. L. (2009). A randomized cross-over study of the quality of cardiopulmonary resuscitation among females performing 30:2 and hands-only cardiopulmonary resuscitation. BMC Nursing, 8, p. 6 .

Valenzuela, T. D., Spaite, D. W., Meislin, H.W., Clark, L. L., Wright, A. L., \& Ewy, G. A. (1993). Emergency vehicle intervals versus collapse-to-CPR and collapse-to-defibrillation intervals: Monitoring emergency medical services system performance in sudden cardiac arrest. Annals of Emergency Medicine, 22(11), pp. 1678-83.

Wang H.E., Mann N.C., Jacobson K.E., Dai M., Mears G., Smyrski K., Yealy D.M. (2013). National characteristics of emergency medical services responses in the united states. Prehospital Emergency Care, 17, pp. 8-14.

Weiss, S., Fullerton, L., Oglesbee, S., Duerden, B., \& Froman, P. (2013). Does ambulance response time influence patient condition among patients with specific medical and trauma emergencies? Southern Medical Journal, 106(3), pp. 230-235. 
Winer, B. J., Brown, D. R., \& Michels, K. M. (1991). Statistical principles in experimental design ( $3^{\text {rd }}$ ed). New York: McGraw-Hill, p. 210.

Wolfe, J. A., Maier, G. W., Newton, J .R. , Glower, D. D., Tyson, G. S., Spratt, J. A., Rankin, J. S., \& Olsen, C. O. (1988). Physiologic determinants of coronary blood flow during external cardiac massage. Journal of Thoracic and Cardiovascular Surgery, 95(3), 523-32. 


\section{APPENDIX A}

\section{Human Subjects Protocol Approval Form \\ HUMAN SUBJECTS PROTOCOL APPROVAL FORM Cal Poly, San Luis Obispo}

All Cal Poly faculty, staff, and student research with human subjects, as well as other research involving human subjects that is conducted at Cal Poly, must be reviewed by the Cal Poly Human Subjects

Committee for the protection of human subjects, the researchers, and the University. Human subjects research is defined as any systematic investigation of living human subjects that is designed to develop or contribute to generalizable knowledge. While the ethical guidelines for research are applicable to classroom activities, demonstrations, and assignments, the Human Subjects Committee does not review classroom activities unless data will be collected and used in a systematic investigation.

Researchers should complete all items on this approval form and submit three copies of it, along with three copies of a research protocol (containing the information detailed in Guidelines for Human Subjects Research Protocol), to the Office of Research and Industry Relations (Debbie Hart, Bldg. 38, Room 154). Please feel free to attach an additional page if your responses to any of the items require more space. Your answers to the items on this form, as well as the research protocol, should be typed. The Committee will make every effort to respond to your submission within two to four weeks. Committee approval should be received prior to contacting prospective subjects and collecting data. Please read carefully Cal Poly's Policy for the Use of Human Subjects in Research prior to completing this application.

1. Date:

January 7, 2014

\section{Title of Research Project:}

The effect of physical fitness on the quality of continuous chest compressions: A comparison between compression rates and professional versus layperson rescuers.

\section{Type of Research:}

Senior project

$\mathrm{X}$ Master's thesis

$\mathrm{X}$ Faculty research

Other:

please explain 


\section{Name(s) of Researcher(s)}

Principal Investigator:

Christopher Cassidy

Department or other affiliation:

Kinesiology Department - Cal Poly University

Phone:

\begin{tabular}{|l|}
\hline (831) 601-6124 \\
\hline
\end{tabular}

Email: $\quad$ ccassidy@calpoly.edu

Position:

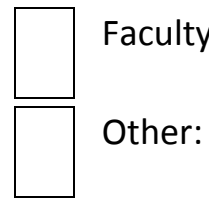

Additional Researcher:

Dr. Steven Craig Davis

Department or other affiliation: Kinesiology

\begin{tabular}{|c|c|c|c|c|c|}
\hline Phone: & & 5) $756-27$ & & Email: & sdavis@calpoly.edu \\
\hline Position: & $x$ & Faculty & & \multicolumn{2}{|c|}{ Student } \\
\hline & & Other: & \multicolumn{3}{|c|}{ please explain } \\
\hline \multicolumn{6}{|c|}{ Additional Researcher: } \\
\hline \multicolumn{6}{|c|}{ Department or other affiliation: } \\
\hline Phone: & & & & Email: & \\
\hline Position: & & Faculty & & \multicolumn{2}{|c|}{ Student } \\
\hline & & Other: & \multicolumn{3}{|c|}{ please explain } \\
\hline
\end{tabular}

Any additional researchers involved in the project should be listed with the descriptive information requested above on a separate sheet. 
5. Faculty Advisor (if applicable)

\begin{tabular}{|c|c|c|c|c|c|}
\hline Name: & \multicolumn{2}{|c|}{ Dr. Steven Craig Davis } & Email: & \multicolumn{2}{|c|}{ sdavis@calpoly.edu } \\
\hline $\begin{array}{l}\text { Departr } \\
\text { affiliation: }\end{array}$ & ent or other & Kinesiology & & Phone: & $\begin{array}{l}(805) 756- \\
2754\end{array}$ \\
\hline
\end{tabular}

Other thesis committee members if the research is a thesis:

\begin{tabular}{|c|c|c|c|c|c|}
\hline Name: & \multicolumn{2}{|c|}{ Dr. Pat Hosegood Martin } & \multirow[t]{2}{*}{ Email: } & \multicolumn{2}{|c|}{ pmartin@calpoly.edu } \\
\hline \multicolumn{2}{|c|}{$\begin{array}{l}\text { Department or other } \\
\text { affiliation: }\end{array}$} & Human Resources & & Phone: & $\begin{array}{l}(805) 756- \\
1151\end{array}$ \\
\hline Name: & \multicolumn{2}{|l|}{ Greg Hall } & Email: & \multicolumn{2}{|c|}{ ghall@calpoly.edu } \\
\hline \multicolumn{2}{|c|}{$\begin{array}{l}\text { Department or other } \\
\text { affiliation: }\end{array}$} & \multicolumn{2}{|l|}{ Kinesiology } & Phone: & $\begin{array}{l}(805) 756- \\
5366\end{array}$ \\
\hline Name: & \multicolumn{2}{|l|}{ Dr. Kellie Green Hall } & Email: & \multicolumn{2}{|c|}{ kghall@calpoly.edu } \\
\hline \multicolumn{2}{|c|}{$\begin{array}{l}\text { Department or other } \\
\text { affiliation: }\end{array}$} & \multicolumn{2}{|l|}{ Kinesiology } & Phone: & $\begin{array}{l}(805) 756- \\
1786\end{array}$ \\
\hline
\end{tabular}

\section{Is there an external funding source for the project:}

Yes, and the source is:

$X \quad$ No

7. Is this a modification of a project previously reviewed by Cal Poly's Human Subjects Committee?

\begin{tabular}{|l|l}
\hline & Yes, and the approximate date of the last review \\
was:
\end{tabular}

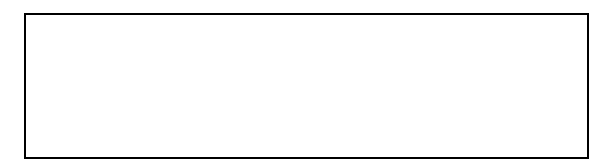




\section{Estimated duration of the project:}

\begin{tabular}{l|l|l|} 
Starting date: & January 01, $2014 \quad$ Completion date: & March 21, 2014 \\
\hline
\end{tabular}

\section{Describe any risks (physical, psychological, social, or economic) that may be involved.}

See Specific Ethical Criterion \#1 in Policy for the Use of Human Subjects in Research for a description of the types of risks.

Risks associated with this study include possible physical complications as a result of the subjects participating in hands-on chest compressions or exercise testing. The continuous compression ratio may prove to be strenuous beyond our subject's capacity to perform.

10. Indicate what measures will be taken to minimize risks. See Specific Ethical Criterion \#1 in Policy for the Use of Human Subjects in Research for a discussion of strategies for minimizing risks.

Medically trained personnel will be present to monitor the subject's state of exertion. A close watch will be administered for possible signs and symptoms that might warrant the stopping of the test.

Experimenters will be watchful for decreased levels of perception or postural signs of fatigue while performing the test.

11. Explain how subjects' confidentiality will be protected. See Specific Ethical Criterion \#5 in Policy for the Use of Human Subjects in Research for a discussion of strategies for minimizing risks.

In order to protect our subjects confidentiality we would include removing face sheets containing identifying information from PAR-Q questionnaire, substituting code numbers for names or other identifiers, limiting the number of individuals with access to data containing identifiers, and storing data in locked cabinets.

Identifiers will be destroyed after the data has been analyzed.

12. Describe any incentives for participation that will be used. See Specific Ethical Criterion \#2 in 
Policy for the Use of Human Subjects in Research for a discussion of the use of incentives in research.

There will be no incentives for subjects involved in this study. Participation in the study, as addressed in the research proposal, is not coerced in any direct or indirect manner. Subjects will not be excluded from participation unless medical screening deems them unable to perform.

13. Will deception of subjects be involved in the research procedures?

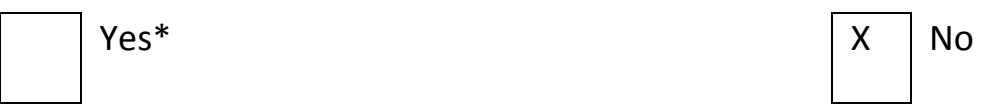

*If so, explain the deception and how it will be handled. See Specific Ethical Criterion \#3 in Policy for the Use of Human Subjects in Research for a discussion of the use of deception in research:

\section{Type of review requested:}

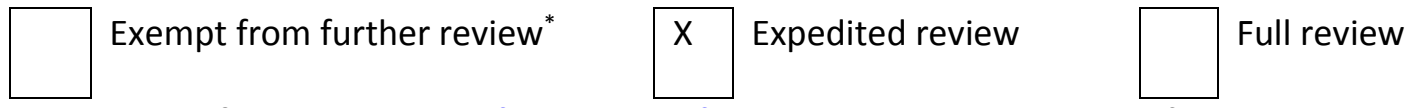

See Types of Review in Policy for the Use of Human Subjects in Research for a discussion of the criteria for exempt, expedited, and full reviews.

*The research protocol submitted for a project presumed to be exempt may be abbreviated but should contain sufficient information to support the conclusion that the project meets the criteria for exemption.

\section{Signatures:}

Your signature below indicates that the information presented in this application (the approval form and research protocol) is accurate and that you have read, understand, and agree to follow the Policy for the Use of Human Subjects in Research. Name of Primary Researcher: Christopher Cassidy Signature: 
Cal Poly Faculty Advisor's Signature (Required if this is student research)

I have reviewed this research proposal which has been prepared by my advisee(s) in accordance with the Guidelines for Obtaining Human Subjects Approval.

Name of Faculty Advisor: Dr. Steven Craig Davis

Signature 


\section{APPENDIX B}

\section{Informed Consent Form}

INFORMED CONSENT TO PARTICIPATE IN A RESEARCH

PROJECT, "The Effect of Physical Fitness on Continuous Chest Compressions: A Comparison Between Compression Rates and Professional Versus Layperson Rescuers."

A research project comparing two different compression rates is being conducted by Christopher Cassidy, a graduate student, and Dr. Steven Craig Davis in the Department of Kinesiology at Cal Poly, San Luis Obispo. The purpose of this study is to investigate how physical fitness affects different compression rates on the quality of compressions during continuous compressions in the layperson versus the professional rescuer.

You are being asked to take part in this study by performing a bench press test, a 3-minute step test, and two continuous external chest compression tests for up to 15 minutes each. During this period, your heart rate, Electrocardiograph (ECG), and Rating of Perceived Exertion (RPE) levels will be recorded along with measures regarding the quality of chest compressions being exhibited. An initial screening process will take place prior to testing whereby you will be asked to complete a Physical Activity Readiness Questionnaire (PAR-Q) and brief targeted history form. If you volunteer to participate, your participation will take approximately 80 minutes. Please be aware that you are not required to participate in this research and you may discontinue your participation at any time without penalty. You also do not have to answer any questions you choose not to answer.

There are possible physical risks associated with participation in this study. Physical complications may occur such as muscle soreness and/or discomfort, injury to the upper extremities or lower back, and associated pain. If you should experience physical complications and you are a Cal Poly student, please be aware that you may contact (805) 756-1211 or visit the Cal Poly Health Center located on University campus at 1 Grand Ave., San Luis Obispo, CA, Building 27. If you are not a Cal Poly student, you may contact your primary care physician for assistance, but you will be responsible for any costs of your medical care.

Your confidentiality will be protected by removing face sheets containing identifying information from questionnaires, substituting code numbers for names or other identifiers, limiting the number of individuals with access to data, and storing data in locked cabinets. The subject list that matches the code number with your identity will be kept in a secure location separate from the data. Your name will not be used in any reports of this research without your permission. Potential benefits associated with the study include adding to the CPR provider knowledge base and helping improve successful resuscitation rates. Your participation in the study will help determine if a certain level of physical fitness is needed to maintain a higher compression rate, and if slower compression rates may be recommended when advanced provider response to a 
cardiac arrest is going to be delayed for more than a few minutes. (If necessary, the following will be added to the consent form. "In addition, if you complete the tests in this study you will be given a \$5 Starbucks or Jamba Juice card," or, "In addition, if you complete the tests in this study your name will be entered into a drawing for an Apple iPAD which will be awarded at the end of the study. The odds of winning are estimated to be approximately 1 in $200 . "$ )

If you have questions regarding this study or would like to be informed of the results when the study is completed, please feel free to contact Christopher Cassidy (ccassidy@calpoly.edu) or Dr. Steven Craig Davis (sdavis@calpoly.edu). If you have questions or concerns regarding the manner in which the study is conducted, you may contact Dr. Dean Wendt, Interim Dean of Research, at (805) 756-1508, dwendt@calpoly.edu.

If you agree to voluntarily participate in this research project as described, please indicate your agreement be signing below. Please keep one copy of this form for your reference, and thank you for you participation in this research.
Signature of Volunteer
Date

Signature of Researcher

Date 
Physical Activity Readiness Questionnaire (PAR-Q)

Physical Activity Readiness Questionnaire - PAR-Q (revised 2002)

\section{PAR-Q \& YOU}

(A Questionnaire for People Aged 15 to 69)

Regular physical activity is fun and healthy, and increasingly more people are starting to become more active every day. Being more active is very safe for most people. However, some people should check with their doctor before they start becoming much more physically active.

If you are planning to become much more physically active than you are now, start by answering the seven questions in the box below. If you are between the ages of 15 and 69, the PAR-Q will tell you if you should check with your doctor before you start. If you are over 69 years of age, and you are not used to being very active, check with your doctor.

Common sense is your best guide when you answer these questions. Please read the questions carefully and answer each one honestly: check YES or NO.

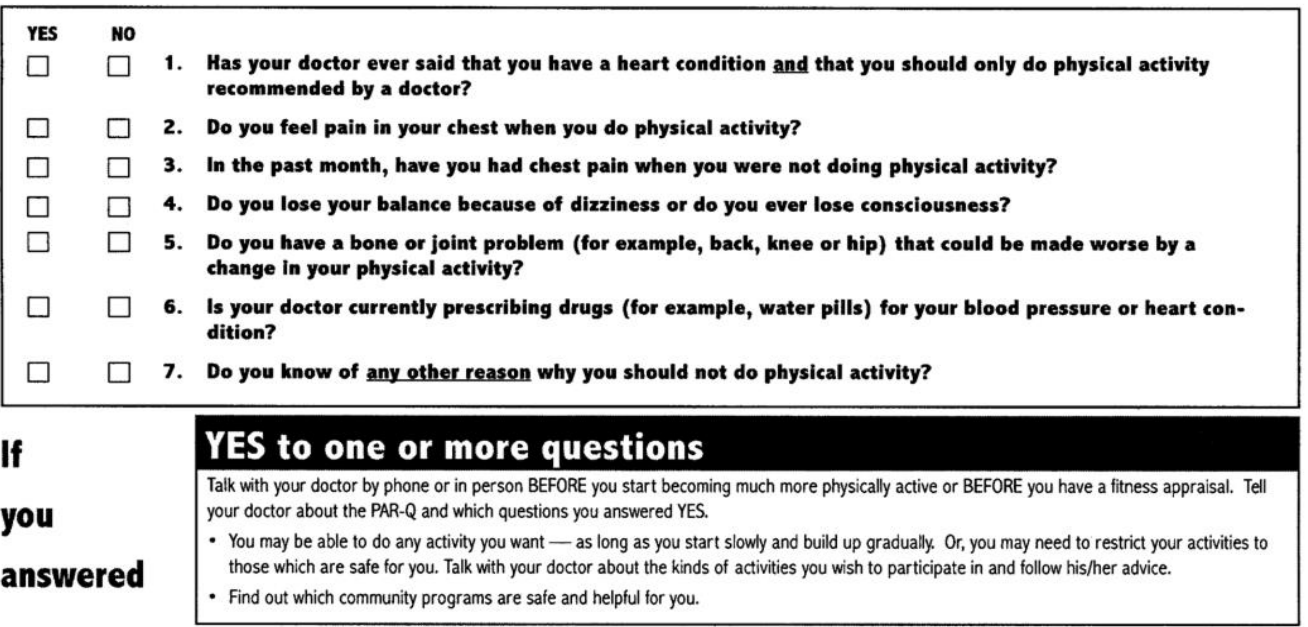

\section{NO to all questions \\ If you answered NO honestly to all PAR-Q questions, you can be reasonably sure that you can: - start becoming much more physically active - begin slowly and build up gradually. This is the safest and easiest way to go. \\ - take part in a fitness appraisal - this is an excellent way to determine your basic fitness so that you can plan the best way for you to live actively. It is also highly recommended that you have your blood pressure evaluated. If your reading is over $144 / 94$, talk with your doctor before you start becoming much more physically active.}

DELAY BECOMING MUCH MORE ACTIVE:

- if you are not feeling well because of a temporary illness such as a cold or a fever - wait until you feel better; or

- if you are or may be pregnant - talk to your doctor before you start becoming more active.

PLEASE NOTE: If your health changes so that you then answer YES to any of the above questions, tell your fitness or health profession Ask whether you should change your physical activity plan.

Informed Use of the PAR-Q: The Canadian Society for Exercise Physiology, Health Canada, and their agents assume no liability for persons who undertake physical activity, and if in doubt after completing Infermed Use of the PAR-Q: The Canadian Society for Exercise Phys
this questionnaire, consult your doctor prior to physical activity

No changes permitted. You are encouraged to photocopy the PAR-Q but only if you use the entire form.

NOTE: If the PAR-Q is being given to a person before he or she participates in a physical activity program or a fitness appraisal, this section may be used for legal or administrative purposes.

"I have read, understood and completed this questionnaire. Any questions I had were answered to my full satisfaction."

NAME

SIGNATURE

SIGNATURE OF PARENT
or GUAROIAN (for participants under the age of majority) \begin{tabular}{|l}
$\begin{array}{l}\text { Note: This physical activity clearance is valid for a maximum of } 12 \text { months from the date it is completed and } \\
\text { becomes invalid if your condition changes so that you would answer } Y E S \text { to any of the seven questions. }\end{array}$ \\
\hline
\end{tabular}

E (c) Canadian Society for Exercise Physiology Supported by: $1+40$ Health Santé

continued on other side... 


\section{APPENDIX D}

\section{YMCA Bench Press Test}

The most widely accepted technique for evaluating strength is probably the one repetition-maximum (1-RM) in which the most weight that can be lifted once through a full range of motion is determined. Because of the increased possibility of injury using a maximal effort technique in untrained individuals, various prediction methods using sub maximal loads have been suggested. The YMCA test was adopted in an attempt to evaluate strength and muscular endurance using a relatively light load.

\section{Equipment}

- Barbell

Men: $80 \mathrm{lbs}(36 \mathrm{~kg})$

Women: 35 lbs $(16 \mathrm{~kg})$

- Bench

- Metronome (set at $60 \mathrm{bpm})$

\section{Procedure}

1. Subject lies supine on bench, knees bent, feet flat on floor

2. Hand barbell to subject

3. Subject grips bar (overhand) at shoulder width

4. Subject presses the bar over the chest fully extending the elbow

5. Subject lowers the bar back to the chest

6. Subject must maintain rhythm with beat of metronome for each phase of movement.

7. 30 repetitions should be completed in one minute.

8. Encourage subject to breathe regularly and not strain during test

9. Stop test when subject no longer can keep pace of metronome

10. Record successful number of repetitions 
NORMS FOR YMCA BENCH PRESS TEST: Total Lifts*

\begin{tabular}{|c|c|c|c|c|c|c|}
\hline \multicolumn{7}{|c|}{ YMCA Bench-Press Test: Total Lifts* } \\
\hline \multirow{2}{*}{$\begin{array}{l}\text { Age (yrs) } \\
\text { Gender }\end{array}$} & \multicolumn{2}{|c|}{$18-25$} & \multicolumn{2}{|c|}{$26-35$} & \multicolumn{2}{|c|}{$36-45$} \\
\hline & M & $\mathbf{F}$ & M & $\mathbf{F}$ & M & $\mathbf{F}$ \\
\hline Excellent & $44-64$ & $42-66$ & 41-61 & $40-62$ & $36-55$ & 33-57 \\
\hline Good & $34-41$ & $30-38$ & $30-37$ & $29-34$ & $26-32$ & $26-30$ \\
\hline Above Average & $29-33$ & $25-28$ & $26-29$ & $24-28$ & $22-25$ & $21-24$ \\
\hline Average & $24-28$ & $20-22$ & $21-24$ & $18-22$ & $18-21$ & $16-20$ \\
\hline Below Average & $20-22$ & $16-18$ & $17-20$ & $14-17$ & $14-17$ & $12-14$ \\
\hline Poor & $13-17$ & $9-13$ & $12-16$ & $9-13$ & $9-12$ & $6-10$ \\
\hline Very Poor & $0-10$ & $0-6$ & $0-9$ & $0-6$ & $0-6$ & $0-4$ \\
\hline Age (yrs) & \multicolumn{2}{|c|}{$46-55$} & \multicolumn{2}{|c|}{ 56-65 } & \multicolumn{2}{|c|}{$\geq 65$} \\
\hline Gender & M & $\mathbf{F}$ & M & $\mathbf{F}$ & M & $\mathbf{F}$ \\
\hline Excellent & $28-47$ & $29-50$ & $24-41$ & $24-42$ & $20-36$ & $18-30$ \\
\hline Good & $21-25$ & $20-24$ & $17-21$ & $17-21$ & $12-16$ & $12-16$ \\
\hline Above Average & $16-20$ & $14-18$ & $12-14$ & $12-14$ & 10 & $8-10$ \\
\hline Average & $12-14$ & $10-13$ & $9-11$ & $8-10$ & $7-8$ & $5-7$ \\
\hline Below Average & $9-11$ & $7-9$ & $5-8$ & $5-6$ & $4-6$ & $3-4$ \\
\hline Poor & $5-8$ & $2-6$ & $2-4$ & $2-4$ & $2-3$ & $0-2$ \\
\hline Very Poor & $0-2$ & $0-1$ & $0-1$ & $0-1$ & $0-1$ & 0 \\
\hline
\end{tabular}



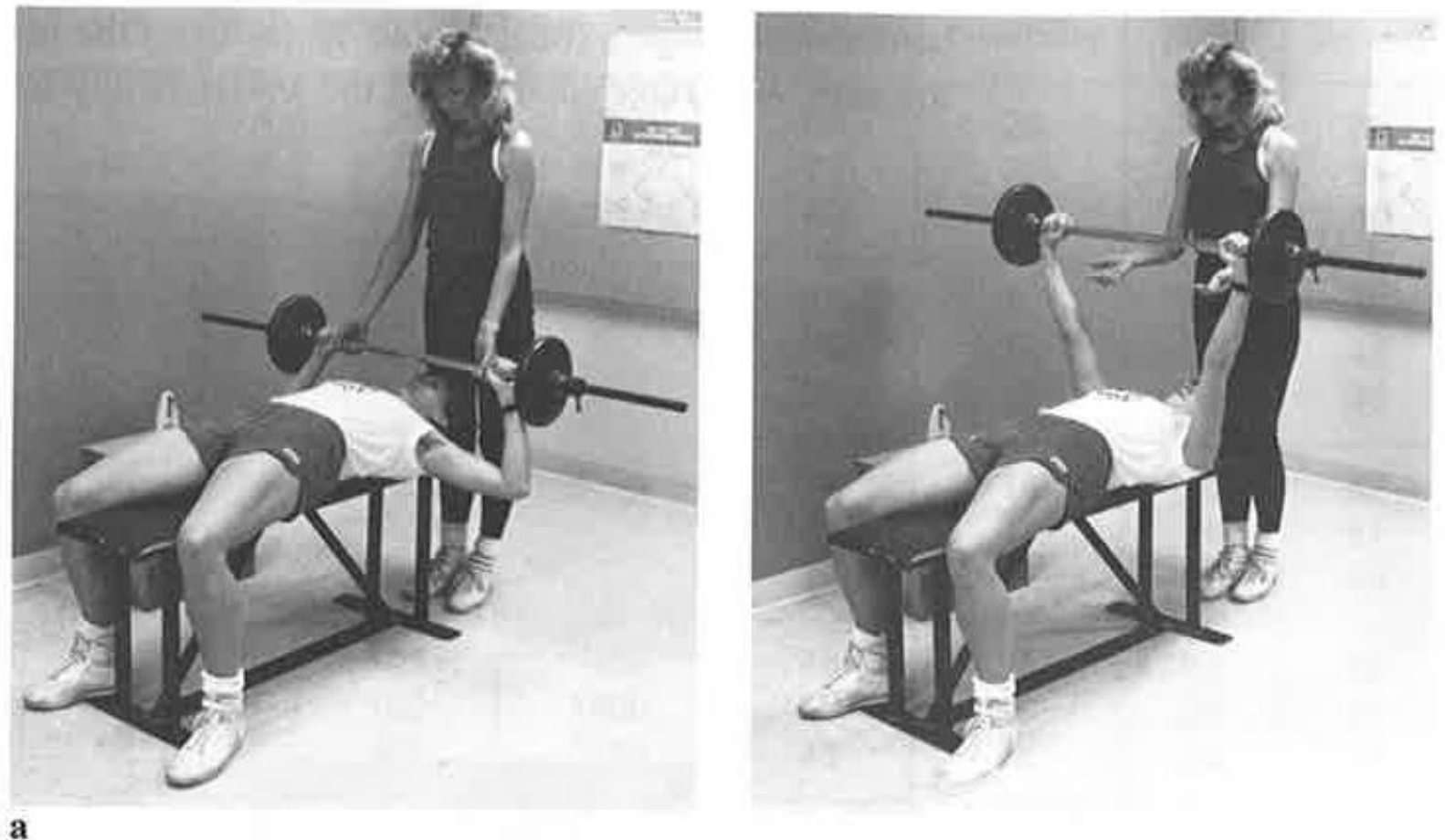


\section{APPENDIX E}

\section{YMCA 3-Minute Step Test}

The YMCA Step test requires the member to step for three minutes at a prescribed stepping cadence of 24 steps per minute. We suggest you orient the member to the testing protocol prior to the test. Allow the member to practice stepping to the prescribed cadence, and that you practice finding their pulse at the end of stepping so they know what to expect. Taking a minute to orient them to these procedures will enhance the reliability of the results.

Required Materials:

12.25 inch step, Stop watch, Metronome

Procedures:

Set the metronome to 96 beats per minute ( 24 steps per minute)

1. Allow the member to practice stepping to the prescribed cadence. Each beat is a step "up" or "down". Note that both feet must come up and down to complete a step, e.g. "upup, downdown"

2. On command, the member should commence stepping as you start the watch.

3. After stepping for three minutes, instruct the member to stop stepping and sit down on the step or in a chair while you find their radial pulse on the inside of their wrist.

4. At 3:05, start counting the heart rate for 60 seconds so that you stop counting at 4:05.

5 . Record the number of beats you counted for the 60 second period.

Important notes:

- The member must remain still and seated during the measurement period following the test.

- Any delay in measuring the heart rate after the test will produce an invalid result and require a retest on another visit. Therefore, be sure to start measuring at 3:05.

\begin{tabular}{|c|c|c|c|c|c|c|}
\hline $\begin{array}{c}\text { Ratings For } \\
\text { Men (age) }\end{array}$ & $\mathbf{1 8 - 2 5}$ & $\mathbf{2 6 - 3 5}$ & $\mathbf{3 6 - 4 5}$ & $\mathbf{4 6 - 5 5}$ & $\mathbf{5 6 - 6 5}$ & $\mathbf{6 5 +}$ \\
\hline Excellent & $50-76$ & $51-76$ & $49-76$ & $56-82$ & $60-77$ & $59-81$ \\
\hline Good & $79-84$ & $79-85$ & $80-88$ & $87-93$ & $86-94$ & $87-92$ \\
\hline Above Average & $88-93$ & $88-94$ & $92-88$ & $95-101$ & $97-100$ & $94-102$ \\
\hline Average & $95-100$ & $96-102$ & $100-105$ & $103-111$ & $103-109$ & $104-110$ \\
\hline Below Average & $102-107$ & $104-110$ & $108-113$ & $113-119$ & $111-117$ & $114-118$ \\
\hline Poor & $111-119$ & $114-121$ & $116-124$ & $121-126$ & $119-128$ & $121-126$ \\
\hline Very Poor & $124-157$ & $126-161$ & $130-163$ & $131-159$ & $131-154$ & $130-151$ \\
\hline
\end{tabular}




\begin{tabular}{|c|c|c|c|c|c|c|}
\hline $\begin{array}{c}\text { Ratings For } \\
\text { Women (age) }\end{array}$ & $\mathbf{1 8 - 2 5}$ & $\mathbf{2 6 - 3 5}$ & $\mathbf{3 6 : 4 5}$ & $\mathbf{4 6 - 5 5}$ & $\mathbf{5 6 - 6 5}$ & $\mathbf{6 5 +}$ \\
\hline Excellent & $52-81$ & $58-80$ & $51-84$ & $63-91$ & $60-92$ & $70-92$ \\
\hline Good & $85-93$ & $85-92$ & $89-96$ & $95-101$ & $97-103$ & $96-101$ \\
\hline Above Average & $96-102$ & $95-101$ & $100-104$ & $104-110$ & $106-111$ & $104-111$ \\
\hline Average & $104-110$ & $104-110$ & $107-112$ & $113-118$ & $113-118$ & $116-121$ \\
\hline Below Average & $113-120$ & $113-119$ & $115-120$ & $120-124$ & $119-127$ & $123-126$ \\
\hline Poor & $122-131$ & $122-129$ & $124-132$ & $126-132$ & $129-135$ & $128-133$ \\
\hline Very Poor & $135-169$ & $134-171$ & $137-169$ & $137-171$ & $141-174$ & $135-155$ \\
\hline
\end{tabular}



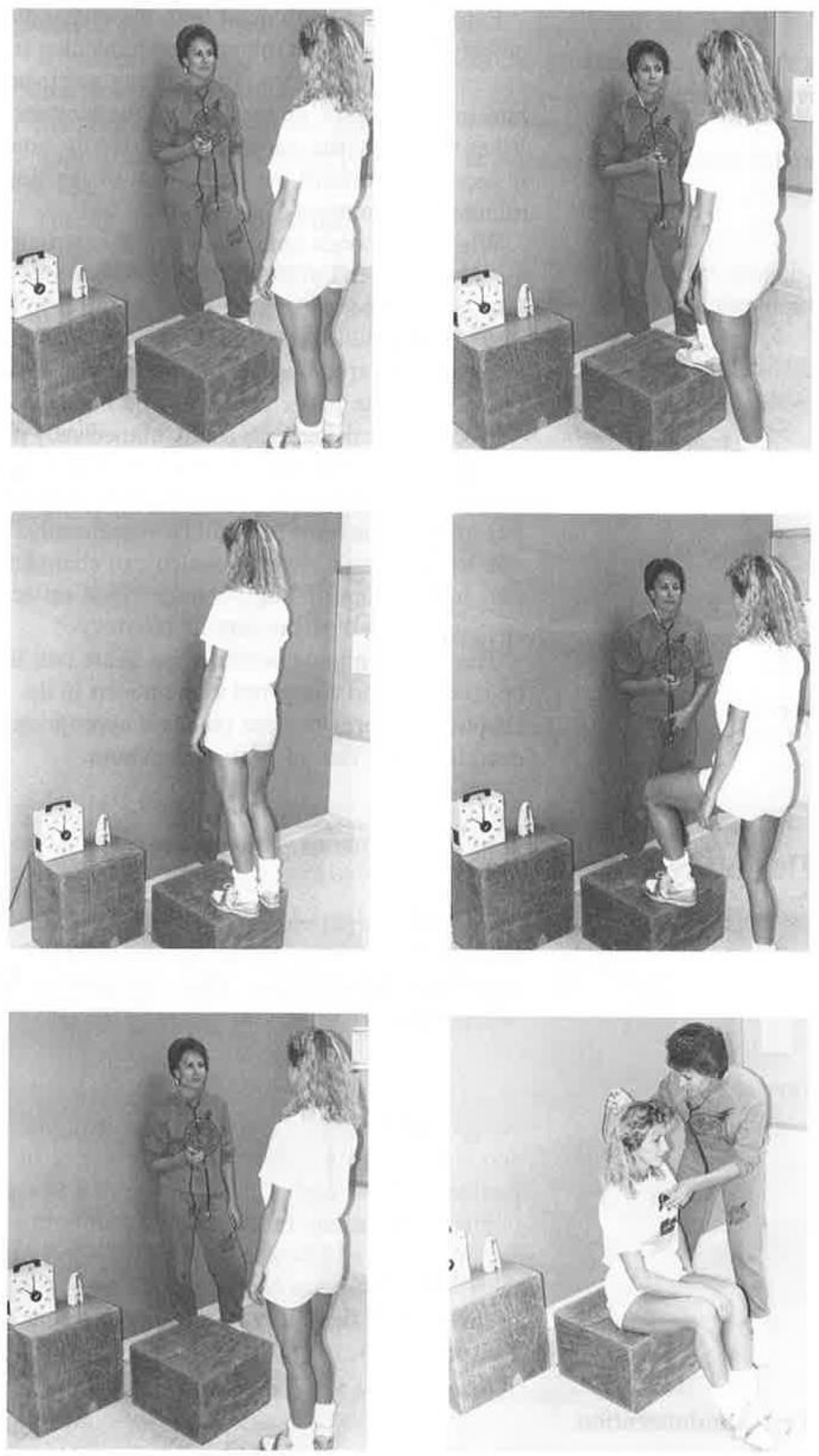


\section{APPENDIX F}

\section{Guidelines for CPR}

American Heart Association (AHA) guidelines for performing chest compressions in CPR are as follows:

1. Put the person on his or her back on a firm surface.

2. Kneel next to the person's neck and shoulders.

3. Place the heel of one hand over the center of the person's chest, between the nipples. Place your other hand on top of the first hand. Keep your elbows straight and position your shoulders directly above your hands.

4. Use your upper body weight (not just your arms) as you push straight down on (compress) the chest at least 2 inches (approximately 5 centimeters). Push hard at a rate of about 100 compressions a minute.

5. If you haven't been trained in CPR, continue chest compressions until there are signs of movement or until emergency medical personnel take over. If you have been trained in CPR, go on to checking the airway and rescue breathing.
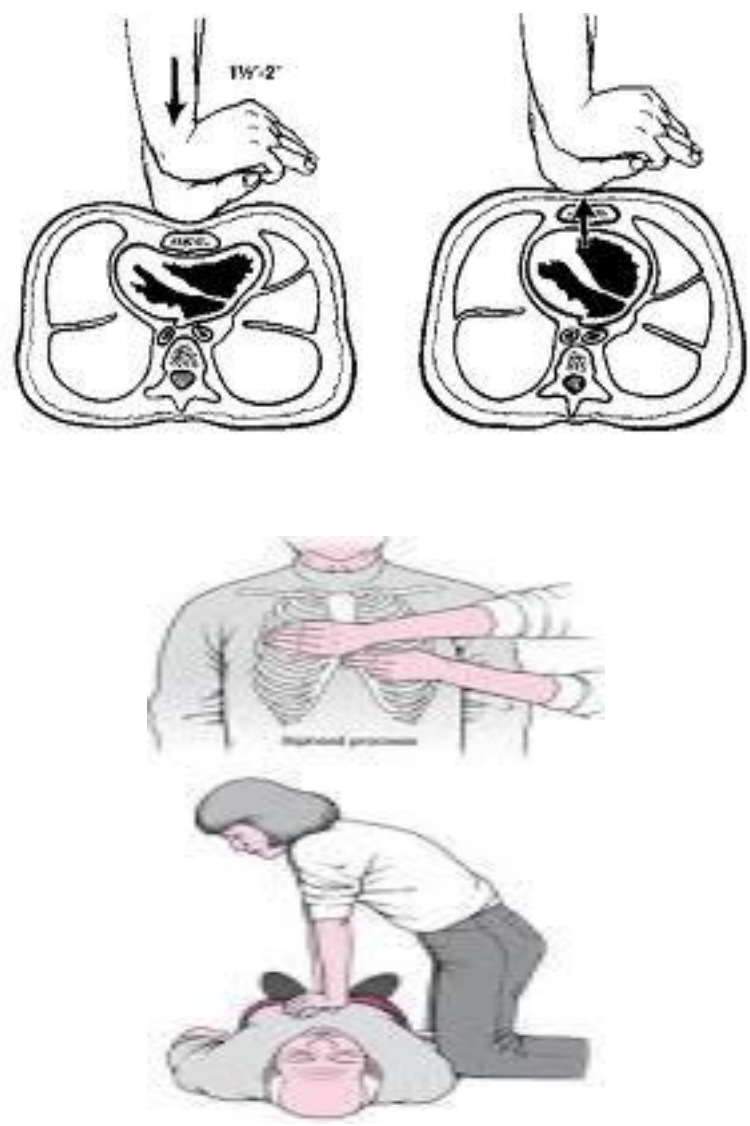